\title{
Hybrid finite compact-WENO schemes for shock calculation
}

\author{
Yiqing Shen* ${ }^{\dagger}$ and Guowei Yang $\ddagger$ \\ LHD, Institute of Mechanics, Chinese Academy of Sciences, Beijing 100080, People's Republic of China
}

\begin{abstract}
SUMMARY
Hybrid finite compact (FC)-WENO schemes are proposed for shock calculations. The two sub-schemes (finite compact difference scheme and WENO scheme) are hybridized by means of the similar treatment as in ENO schemes. The hybrid schemes have the advantages of FC and WENO schemes. One is that they possess the merit of the finite compact difference scheme, which requires only bi-diagonal matrix inversion and can apply the known high-resolution flux to obtain high-performance numerical flux function; another is that they have the high-resolution property of WENO scheme for shock capturing. The numerical results show that FC-WENO schemes have better resolution properties than both FC-ENO schemes and WENO schemes. In addition, some comparisons of FC-ENO and artificial compression method (ACM) filter scheme of Yee et al. are also given. Copyright (c) 2006 John Wiley \& Sons, Ltd.
\end{abstract}

Received 16 May 2005; Revised 26 April 2006; Accepted 26 April 2006

KEY WORDS: finite compact difference scheme; ENO scheme; WENO scheme; ACM filter scheme; shock calculation

\section{INTRODUCTION}

In many practical aerodynamic problems, the flow field often involves the complicated regions that contain both shocks and smooth structures. For the numerical simulation of these problems, it is required that the schemes have the capability of shock capturing and fine-scale feature capturing.

One class of efficient shock-capturing schemes are TVD [1], ENO [2,3] and WENO [4,5] schemes. ENO schemes overcome the well-known order-degeneracy phenomenon of the TVD schemes at critical points, and can obtain uniformly high-order accuracy right up to the discontinuity. WENO schemes have the advantage over the ENO ones to yield higher-order accuracy

\footnotetext{
${ }^{*}$ Correspondence to: Yiqing Shen, LHD, Institute of Mechanics, Chinese Academy of Sciences, Beijing 100080, People's Republic of China.

†E-mail: yqshen@imech.ac.cn

${ }^{\ddagger}$ E-mail: gwyang@imech.ac.cn

Contract/grant sponsor: National Natural Science Foundation of China; contract/grant numbers: 10402043, 10372106
} 
for the same stencil size. The ENO and WENO schemes can resolve flow features accurately and robustly, however, they give slightly dissipative solutions [6]. There are lots of works to improve the performance of ENO schemes, such as Yee et al. [7] developed a new low-dissipative highorder shock-capturing scheme (for simplicity, in the article, the scheme is referred to as ACM filter scheme as in Reference [8] or ACM) that is composed of the high-order spatial and temporal base scheme and the filter (the numerical dissipation); Wang and Chen [9] proposed modification of the WENO smoothness measures and developed an optimized WENO scheme, which achieves better resolution of high wavenumbers. Balsara and Shu [10] proposed the MPWENO (monotonicity-preserving WENO) scheme that is up to 11th-order accurate in space, Kim and Kwon [6] proposed a hybrid central-WENO scheme in which the sixth-order central difference scheme and the WENO-5 are applied. Jiang [11] has proposed the dispersion-controlled dissipative scheme.

Compact schemes have become popular due to their high-order accuracy and small stencils [12], but for the problems involving discontinuities, they may produce oscillations whose amplitude does not attenuate even using denser grid. In recent years, there has been an increased interest in developing high-order accurate compact schemes to adapt to capturing shock. Cockburn and Shu [13] presented the idea of TVDM (total variation diminishing in the means) to develop nonlinearly stable compact schemes, which have uniform high-order accuracy even at local extremum point. Ravichandran [14] developed further this kind of scheme to TVD scheme, which uses a wider class of compact upwind operators and together with the split fluxes of the KFVS (kinetic flux vector splitting) scheme to obtain high-order semi-discretizations of two-dimensional Euler equations in general coordinates. Deng and Zhang [15] developed an algorithm whereby the resolution properties of WENO schemes are improved by introducing compact approximations for pointwise derivatives rather than explicit ones. Zhu and Chan [16] proposed a third-order upwind compact scheme, in the region where pressure gradient is large, group velocity of the scheme can be controlled [17] to eliminate the numerical oscillations. An alternative effective approach is to develop so-called hybrid methods in which the non-oscillatory shock-capturing schemes are only used locally near the discontinuities and the compact schemes are used in smooth regions. Adams and Shariff [18] developed hybrid schemes that rely on the coupling of a non-conservative compact upwind scheme with a shock-capturing ENO scheme which operates on the discontinuity region. Pirozzoli [19] improved in several aspects of this approach, proposed the conservative hybrid compact-WENO schemes. Ren et al. [20] presented a characteristic-wise hybrid compactWENO scheme, which can be considered as the weighted average of two sub-schemes: the conservative compact scheme proposed by Pirozzoli [19] and the WENO proposed by Jiang and Shu [5].

For many compact schemes, it is necessary to solve the inversion of a penta-diagonal matrix [18] or tri-diagonal matrix [13,14, 19, 20]. In addition, the performance of those hybrid schemes [18-20] rely on the problem-dependent threshold value $r_{c}$, which are used to decide where the numerical flux of the ENO/WENO may be used. From the point of view of avoiding the problemdependent parameter, Ravichandran's method [14] is successful. In order to illustrate the method it is convenient to consider the scalar conservative hyperbolic equation

$$
\begin{aligned}
\frac{\partial u}{\partial t}+\frac{\partial f}{\partial x} & =0 \\
u(x, 0) & =u^{0}(x)
\end{aligned}
$$


where $f(u)$ is the flux function and can be split into two parts, i.e. $f(u)=f^{+}(u)+f^{-}(u)$ with $\mathrm{d} f^{+}(u) / \mathrm{d} u \geqslant 0$ and $\mathrm{d} f^{-}(u) / \mathrm{d} u \leqslant 0$ and $\mathrm{d} f^{-}(u) / \mathrm{d} u \leqslant 0$. The semi-discrete conservative difference scheme of (1) can be written as follows:

$$
\frac{\mathrm{d} u_{j}}{\mathrm{~d} t}+\frac{1}{\Delta x}\left(h_{j+1 / 2}-h_{j-1 / 2}\right)=0
$$

where the numerical flux $h_{j+1 / 2}=h_{j+1 / 2}^{+}+h_{j+1 / 2}^{-}$.

The Ravichandran's method [14] can be divided into two steps. Firstly, the compact flux is solved by using the following equations:

$$
\begin{aligned}
& \alpha^{+} \hat{h}_{j-1 / 2}^{+}+\beta^{+} \hat{h}_{j+1 / 2}^{+}+\gamma^{+} \hat{h}_{j+3 / 2}^{+}=\sum_{l=n_{1}}^{n_{2}} a_{l}^{+} f_{j+l}^{+} \\
& \gamma^{-} \hat{h}_{j-1 / 2}^{-}+\beta^{-} \hat{h}_{j+1 / 2}^{-}+\alpha^{-} \hat{h}_{j+3 / 2}^{-}=\sum_{l=n_{3}}^{n_{4}} a_{l}^{-} f_{j+l}^{-}
\end{aligned}
$$

the final numerical flux functions defined as

$$
h_{j+1 / 2}^{+}=f_{j}^{+}+\mathrm{d} f_{j+1 / 2}^{+(m)}, \quad h_{j+1 / 2}^{-}=f_{j+1}^{-}-\mathrm{d} f_{j+1 / 2}^{-(m)}
$$

where $\quad \mathrm{d} f_{j+1 / 2}^{+(m)}=m m\left(\mathrm{~d} f_{j+1 / 2}^{+}, \Delta_{+} f_{j}^{+}, \Delta_{+} f_{j-1}^{+}\right), \quad \mathrm{d} f_{j+1 / 2}^{-(m)}=m m\left(\mathrm{~d} f_{j+1 / 2}^{-}, \Delta_{+} f_{j}^{-}, \Delta_{+} f_{j+1}^{-}\right)$, $\mathrm{d} f_{j+1 / 2}^{+}=\hat{h}_{j+1 / 2}^{+}-f_{j}^{+}, \mathrm{d} f_{j+1 / 2}^{-}=f_{j+1}^{-}-\hat{h}_{j+1 / 2}^{-}, \Delta_{+} f_{j}^{ \pm}=f_{j+1}^{ \pm}-f_{j}^{ \pm}, n_{1}\left(n_{3}\right)$ and $n_{2}\left(n_{4}\right)$ are start- and end-location of $f_{j+l}$ in different schemes. Parameters $\alpha^{ \pm}, \beta^{ \pm}, \gamma^{ \pm}$and $a_{l}^{ \pm}$are decided by the requirement of different accuracy and property of the scheme, for example, for the thirdorder upwind compact scheme [21], they are $\alpha^{ \pm}=5 / 12, \beta^{ \pm}=8 / 12, \gamma^{ \pm}=-1 / 12, a_{0}^{+}=a_{1}^{-}=1$. $m m\left(a_{1}, a_{2}, a_{3}\right)$ is the limiter function and defined as

$$
m m\left(a_{1}, \ldots, a_{k}\right)= \begin{cases}s \min _{1 \leqslant i \leqslant k}\left|a_{i}\right| & \text { if } \operatorname{sign}\left(a_{1}\right)=\cdots=\operatorname{sign}\left(a_{k}\right)=s \\ 0 & \text { otherwise }\end{cases}
$$

In Reference [22], we presented the idea of finite compact (FC) difference schemes in which, instead of Equation (3), the following equations are used:

$$
\begin{aligned}
& \alpha^{+} h_{j-1 / 2}^{+}+\beta^{+} \hat{h}_{j+1 / 2}^{+}=\sum_{l=n_{1}}^{n_{2}} a_{l}^{+} f_{j+l}^{+} \\
& \beta^{-} \hat{h}_{j+1 / 2}^{-}+\alpha^{-} h_{j+3 / 2}^{-}=\sum_{l=n_{3}}^{n_{4}} a_{l}^{-} f_{j+l}^{-}
\end{aligned}
$$

The fluxes $h_{j-1 / 2}^{+}$and $h_{j+3 / 2}^{-}$can be defined as Equation (4) or other ways. With different limiter functions, a third-order accurate FC-TVD scheme, a third-order accurate FC-ENO scheme and a fifth-order accurate FC-ENO scheme were constructed in Reference [22].

Equation (6) has three advantages over Equation (3):

(1) If the boundary fluxes $\hat{h}_{-1 / 2}^{+}$and $\hat{h}_{N+1 / 2}^{-}$are given, $\hat{h}_{j+1 / 2}^{ \pm}$can be solved explicitly, so tri-diagonal matrix inversion of Equation (3) is avoided. 
(2) The fluxes $h_{j-1 / 2}^{+}$and $h_{j+3 / 2}^{-}$having special TVD or ENO characteristics can be constructed in advance, they may not be $\hat{h}_{j-1 / 2}^{+}$and $\hat{h}_{j+3 / 2}^{-}$in Equation (3). This property results in, as the solution contains discontinuities, more accurate and better resolution solution than Equation (3) [14]. Notice that the schemes with a three-point stencil of implicit operator (e.g. Equation (3) with $\alpha^{ \pm} \neq 0, \gamma^{ \pm} \neq 0$ ) cannot use the known fluxes $h_{j-1 / 2}^{+}$and $h_{j+3 / 2}^{-}$to solve $\hat{h}_{j+1 / 2}^{+}$and $\hat{h}_{j+1 / 2}^{-}$.

(3) In Equation (6), the limited (high-resolution) fluxes $h_{j-1 / 2}^{+}$and $h_{j+3 / 2}^{-}$are used at the points in a discontinuity, and the coefficients of $h_{j-1 / 2}^{+}$and $h_{j+3 / 2}^{-}$are less than one, i.e. $\alpha^{ \pm} / \beta^{ \pm}<1$. So, in the region away from the discontinuity, the effect of the limited fluxes on the accuracy become smaller and smaller and Equation (6) can retain its higher order of accuracy.

In Reference [19], it is shown that the overall performance of hybrid schemes is critically affected by the order of accuracy of the shock-capturing scheme that is employed. Although WENO schemes are simulating the base ENO schemes around discontinuities, numerous tests [5] show that the third-order WENO scheme is comparable to the third-order ENO schemes, instead of the base second-order ENO scheme, and the fifth-order WENO scheme is comparable to the fourth-order ENO scheme. WENO schemes therefore represent a better candidate than ENO schemes. In this paper, the WENO fluxes are applied to instead of the TVD/ENO fluxes used in FC-ENO schemes, the hybrid finite compact-WENO methods are proposed.

The boundary flux formulas proposed in Reference [22] will be implemented. The third-order formulas are

$$
\begin{aligned}
h_{-1 / 2}^{+} & =\frac{1}{3} f_{-1}^{+}+\frac{5}{6} f_{0}^{+}-\frac{1}{6} f_{1}^{+} \\
h_{N+1 / 2}^{-} & =-\frac{1}{6} f_{N-1}^{-}+\frac{5}{6} f_{N}^{-}+\frac{1}{3} f_{N+1}^{-}
\end{aligned}
$$

and the fifth-order formulas are

$$
\begin{aligned}
h_{-1 / 2}^{+} & =\frac{1}{60}\left(-3 f_{-2}^{+}+27 f_{-1}^{+}+47 f_{0}^{+}-13 f_{1}^{+}+2 f_{2}^{+}\right) \\
h_{N+1 / 2}^{-} & =\frac{1}{60}\left(2 f_{N-2}^{-}-13 f_{N-1}^{-}+47 f_{N}^{-}+27 f_{N+1}^{-}-3 f_{N+2}^{-}\right.
\end{aligned}
$$

For the time discretization, the third-order TVD Runge-Kutta method [3] and fourth-order Runge-Kutta method are used to discretize Equation (2) with spatial third-order and fifth-order approximation, respectively.

The drawback of the parallelization unfriendliness of compact schemes makes the FC-schemes extremely CPU intensive under parallel dominated supercomputers. The artificial compression method (ACM) filter scheme with different filter proposed by Yee et al. [7, 8, 23, 24] requires a similar CPU as a second-order TVD scheme and yet achieves higher accuracy than fifth-order WENO scheme.

The ACM schemes use spatially high-order compact or non-compact central base scheme with the product of flow sensors and the dissipative portion of TVD, MUSCL or 5th-WENO scheme as a nonlinear filter. For Equation (1), the filter numerical fluxes can be written as

$$
\widetilde{F}_{j+1 / 2}=\omega_{j+1 / 2} F_{j+1 / 2}^{*}
$$


where $F_{j+1 / 2}^{*}$ is the dissipative portion of shock-capturing scheme, a form of the ACM sensor $\omega_{j+1 / 2}$ proposed in Reference [7] is $\omega_{j+1 / 2}=k \max \left(\theta_{j}, \theta_{j+1}\right)$, where the parameter $k$ is problem dependent,

$$
\theta_{j}=\left|\frac{\left|\Delta u_{j+1 / 2}\right|-\left|\Delta u_{j-1 / 2}\right|}{\left|\Delta u_{j+1 / 2}\right|+\left|\Delta u_{j-1 / 2}\right|+\varepsilon}\right|
$$

$\varepsilon$ is a positive real number to avoid divided by zero. The wavelet-based flow sensors can be referred in References [8,23]. With the wavelet sensor, the ACM can be improved.

As mentioned in Reference [8], the nonlinear filter dissipation comes from a second-order accurate TVD method, if no switching were used the order of accuracy would have been reduced to second order. The switch in the ACM method is of order $\Delta x$ when data are smooth, and will therefore give formal order of accuracy three. In practice, the coefficient $k$ is often taken small, so that the constant in front of the third-order error term is very small.

In this paper, we will show the comparison of the FC-WENO schemes and the ACM schemes for some examples. In the computation, if the spatial fourth-order central scheme is applied, the dissipative portion of third-order WENO scheme is taken as a nonlinear filter, we denote it by CEN4+WENO3fi; if the spatial sixth-order central scheme is applied, the dissipative portion of fifth-order WENO scheme is taken as a nonlinear filter, we denote it by CEN6+WENO5fi.

\section{HYBRID FINITE COMPACT WENO SCHEMES}

\subsection{Relations between FC-TVD/ENO schemes and ENO schemes}

Firstly, the coefficients of the third-order [12,25] and fifth-order schemes [26] in Equation (6) are listed in Table I. Here only the positive flux $\hat{h}_{j+1 / 2}^{+}$will be discussed. The coefficients of $f_{j+l}^{-}$in the negative flux $h_{j+1 / 2}^{-}$are symmetric with respect to $x_{j+1 / 2}$ and are not shown here.

The limiter function $m m\left(a_{1}, a_{2}, a_{3}\right)$ used in FC-TVD [22] can be given as

$$
m m\left(a_{1}, a_{2}, a_{3}\right)= \begin{cases}a_{1} & \text { if } \operatorname{sign}\left(a_{1}\right)=\cdots=\operatorname{sign}\left(a_{3}\right) \text { and }\left|a_{1}\right|=\min \left(\left|a_{1}\right|,\left|a_{2}\right|,\left|a_{3}\right|\right) \\ a_{2} / 2 & \text { if } \operatorname{sign}\left(a_{1}\right)=\cdots=\operatorname{sign}\left(a_{3}\right) \text { and }\left|a_{2}\right|=\min \left(\left|a_{1}\right|,\left|a_{2}\right|,\left|a_{3}\right|\right) \\ a_{3} / 2 & \text { if } \operatorname{sign}\left(a_{1}\right)=\cdots=\operatorname{sign}\left(a_{3}\right) \text { and }\left|a_{3}\right|=\min \left(\left|a_{1}\right|,\left|a_{2}\right|,\left|a_{3}\right|\right) \\ 0 & \text { otherwise }\end{cases}
$$

We can find that if the second or third value of the function $m m\left(a_{1}, a_{2}, a_{3}\right)$ is picked, the numerical flux function $h_{j+1 / 2}^{+}$(Equation (4)) becomes the second-order ENO scheme:

$$
h_{j+1 / 2}^{+}=q_{k}^{r}\left(f_{j+k-r+1}^{+}, \ldots, f_{j+k}^{+}\right)
$$

Table I. The coefficients of the third-order and fifth-order schemes in Equation (6).

\begin{tabular}{lrrrrrrr}
\hline Order & $\alpha$ & $\beta$ & $a_{-2}$ & $a_{-1}$ & $a_{0}$ & $a_{1}$ & $a_{2}$ \\
\hline Third & 2 & 4 & - & - & 5 & 1 & - \\
Fifth & 24 & 36 & - & 3 & 47 & 11 & -1 \\
\hline
\end{tabular}


Table II. Coefficients $a_{k, l}^{r}$ with $r=2$.

\begin{tabular}{cccc}
\hline$r$ & $k$ & $l=0$ & $l=1$ \\
\hline 2 & 0 & $-1 / 2$ & $3 / 2$ \\
& 1 & $1 / 2$ & $1 / 2$ \\
\hline
\end{tabular}

where $r=2, k=0$ or $k=1 . q_{k}^{r}\left(g_{0}, \ldots, g_{r-1}\right)=\sum_{l=0}^{r-1} a_{k, l}^{r} g_{l}, a_{k, l}^{r}$ are the coefficients of second ENO scheme concluded in Reference [5], here are listed in Table II.

The numerical flux function $h_{j+1 / 2}^{+}$of the fifth-order accurate FC-ENO scheme can be given as follows:

$$
h_{j+1 / 2}^{+}=f_{j}^{+}+\frac{1}{2} m\left(\Delta_{+} f_{j}^{+}, \Delta_{+} f_{j-1}^{+}\right)+D f_{j+1 / 2}^{+(m)}
$$

where

$$
\begin{aligned}
m\left(a_{1}, a_{2}\right) & = \begin{cases}a_{1} & \text { if }\left|a_{1}\right| \leqslant\left|a_{2}\right| \\
a_{2} & \text { otherwise }\end{cases} \\
D f_{j+1 / 2}^{+(m)} & = \begin{cases}m m 1\left(D f_{j+1 / 2}^{+}, D_{j}^{+}, D_{j+1}^{+}\right) & \text {if }\left|\Delta_{+} f_{j}^{+}\right| \leqslant\left|\Delta_{+} f_{j-1}^{+}\right| \\
m m 2\left(D f_{j+1 / 2}^{+}, D_{j}^{+}, D_{j-1}^{+}\right) & \text {otherwise }\end{cases} \\
m m 1\left(a_{1}, a_{2}, a_{3}\right) & = \begin{cases}a_{1} & \text { if }\left|a_{1}\right|=\min \left(\left|a_{1}\right|,\left|a_{2}\right|,\left|a_{3}\right|\right) \\
-a_{i} / 6 & \text { otherwise }\left|a_{i}\right|=\min \left(\left|a_{1}\right|,\left|a_{2}\right|,\left|a_{3}\right|\right)\end{cases} \\
m m 2\left(a_{1}, a_{2}, a_{3}\right) & = \begin{cases}a_{1} & \text { if }\left|a_{1}\right|=\min \left(\left|a_{1}\right|,\left|a_{2}\right|,\left|a_{3}\right|\right) \\
a_{i} / 3 & \text { otherwise and }\left|a_{i}\right|=\min \left(\left|a_{1}\right|,\left|a_{2}\right|,\left|a_{3}\right|\right)\end{cases}
\end{aligned}
$$

where

$$
\begin{gathered}
D_{j}^{ \pm}=\Delta_{+} f_{j}^{ \pm}-\Delta_{+} f_{j-1}^{ \pm} \\
D f_{j+1 / 2}^{+}=\hat{h}_{j+1 / 2}^{+}-f_{j}^{+}-\frac{1}{2} m\left(\Delta_{+} f_{j}^{+}, \Delta_{+} f_{j-1}^{+}\right) \\
D f_{j+1 / 2}^{-}=\hat{h}_{j+1 / 2}^{-}-f_{j+1}^{-}+\frac{1}{2} m\left(\Delta_{+} f_{j+1}^{-}, \Delta_{+} f_{j}^{-}\right)
\end{gathered}
$$

we can find that if the second or third values of the function $m m 1\left(a_{1}, a_{2}, a_{3}\right)$ or $m m 2\left(a_{1}, a_{2}, a_{3}\right)$ is picked, the flux $h_{j+1 / 2}^{+}$(Equation (11)) becomes the third-order ENO scheme,

$$
h_{j+1 / 2}^{+}=q_{k}^{r}\left(f_{j+k-r+1}^{+}, \ldots, f_{j+k}^{+}\right)
$$

where $r=3, k=0, k=1$ or $k=2 . q_{k}^{r}\left(g_{0}, \ldots, g_{r-1}\right)=\sum_{l=0}^{r-1} a_{k, l}^{r} g_{l}$, the coefficients $a_{k, l}^{r}$ of thirdorder ENO scheme are listed in Table III (see Reference [5]). 
Table III. Coefficients $a_{k, l}^{r}$ with $r=3$.

\begin{tabular}{ccccc}
\hline$r$ & $k$ & $l=0$ & $l=1$ & $l=2$ \\
\hline & 0 & $1 / 3$ & $-7 / 6$ & $11 / 6$ \\
3 & 1 & $-1 / 6$ & $5 / 6$ & $1 / 3$ \\
& 2 & $1 / 3$ & $5 / 6$ & $-1 / 6$ \\
\hline
\end{tabular}

Table IV. Optimal weights $C_{k}^{r}$.

\begin{tabular}{lccc}
\hline$C_{k}^{r}$ & $k=0$ & $k=1$ & $k=2$ \\
\hline$r=2$ & $1 / 3$ & $2 / 3$ & - \\
$r=3$ & $1 / 10$ & $6 / 10$ & $3 / 10$ \\
\hline
\end{tabular}

\subsection{The resolution properties of finite compact schemes and WENO schemes}

The detailed description of the WENO schemes can be referred to Reference [5]. For completeness, the numerical flux functions of the WENO schemes are also given as follows. In the same way,

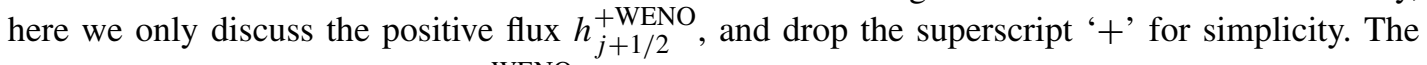
formulas for the negative flux $h_{j+1 / 2}^{- \text {WENO }}$ are symmetric with respect to $x_{j+1 / 2}$.

$$
h_{j+1 / 2}^{\mathrm{WENO}}=\sum_{k=0}^{r-1} \omega_{k} q_{k}^{r}\left(f_{j+k-r+1}, \ldots, f_{j+k}\right)
$$

where the weight $\omega_{k}$ is defined by

$$
\omega_{k}=\frac{\alpha_{k}}{\alpha_{0}+\cdots+\alpha_{r-1}} \quad \text { and } \quad \alpha_{k}=\frac{C_{k}^{r}}{\left(\varepsilon+I S_{k}\right)^{2}}, \quad k=0,1, \ldots, r-1
$$

$C_{k}^{r}$ is the optimal weight, and is given in Table IV. $I S_{k}$ is the smoothness measurement and $\varepsilon$ is a positive real number to avoid divided by zero. When $r=2, I S_{k}$ is computed by

$$
I S_{0}=\left(f_{j}-f_{j-1}\right)^{2}, \quad I S_{1}=\left(f_{j+1}-f_{j}\right)^{2}
$$

When $r=3, I S_{k}$ is computed by

$$
\begin{gathered}
I S_{0}=\frac{13}{12}\left(f_{j-2}-2 f_{j-1}+f_{j}\right)^{2}+\frac{1}{4}\left(f_{j-2}-4 f_{j-1}+3 f_{j}\right)^{2} \\
I S_{1}=\frac{13}{12}\left(f_{j-1}-2 f_{j}+f_{j+1}\right)^{2}+\frac{1}{4}\left(f_{j-1}-3 f_{j+1}\right)^{2} \\
I S_{2}=\frac{13}{12}\left(f_{j}-2 f_{j+1}+f_{j+2}\right)^{2}+\frac{1}{4}\left(3 f_{j}-4 f_{j+1}+f_{j+2}\right)^{2}
\end{gathered}
$$

In order to investigate the resolution properties of the finite compact difference schemes and the WENO schemes, we assume $f=a u$ and $a>0$ in Equation (1) with the initial condition $u(x, 0)=\mathrm{e}^{\mathrm{i} k x}$. The exact solution of Equation (1) is

$$
u(x, t)=\mathrm{e}^{\mathrm{i} k(x-a t)}
$$

and the solution of different difference schemes can be obtained as

$$
u\left(x_{j}, t\right)=\mathrm{e}^{-k_{r} a t / \Delta x} \mathrm{e}^{\mathrm{i} k\left(x_{j}-k_{i} a t / k \Delta x\right)}
$$


It is easy to obtain the formulas of different scheme as follows:

$$
\begin{gathered}
k_{i}^{3-\mathrm{FC}}=\frac{\sin (\alpha)[8+\cos (\alpha)]}{5+4 \cos (\alpha)}, \quad k_{r}^{3-\mathrm{FC}}=\frac{[\cos (\alpha)-1]^{2}}{5+4 \cos (\alpha)} \\
k_{i}^{5-\mathrm{FC}}=\frac{\sin (\alpha)\left[62+15 \cos (\alpha)-2 \cos ^{2}(\alpha)\right]}{39+36 \cos (\alpha)}, \quad k_{r}^{5-\mathrm{FC}}=\frac{-2[\cos (\alpha)-1]^{3}}{39+36 \cos (\alpha)} \\
k_{i}^{\mathrm{WENO}-3}=\frac{1}{6}[-\sin (2 \alpha)+8 \sin (\alpha)], \quad k_{r}^{\mathrm{WENO}-3}=\frac{1}{6}[\cos (2 \alpha)-4 \cos (\alpha)+3] \\
k_{i}^{\mathrm{WENO}-5}=\frac{1}{30}[\sin (3 \alpha)-9 \sin (2 \alpha)+45 \sin (\alpha)] \\
k_{r}^{\mathrm{WENO}-5}=\frac{1}{30}[-\cos (3 \alpha)+6 \cos (2 \alpha)-15 \cos (\alpha)+10]
\end{gathered}
$$

where $\alpha(\alpha=k \Delta x)$ is the scaled wave number. The superscript of $k_{i}$ and $k_{r}$ denotes the different schemes, for example, 3-FC and WENO-3 represent the third-order finite compact difference scheme and the third-order WENO scheme, respectively.

The variations of $k_{i}$ and $k_{r}$ of the FC-schemes, Equation (6), and the WENO-schemes, Equation (13), versus scaled wave number $\alpha$ is given in Figure 1. The figure shows that the FC-schemes yield better resolution properties than the WENO schemes. The dissipation error of 3-FC scheme is larger than the WENO-3 only if $\alpha>\alpha_{c 1}\left(\cos \left(\alpha_{c 1}\right)=-1 / 2\right)$, the similar behaviour of 5-FC scheme occurs
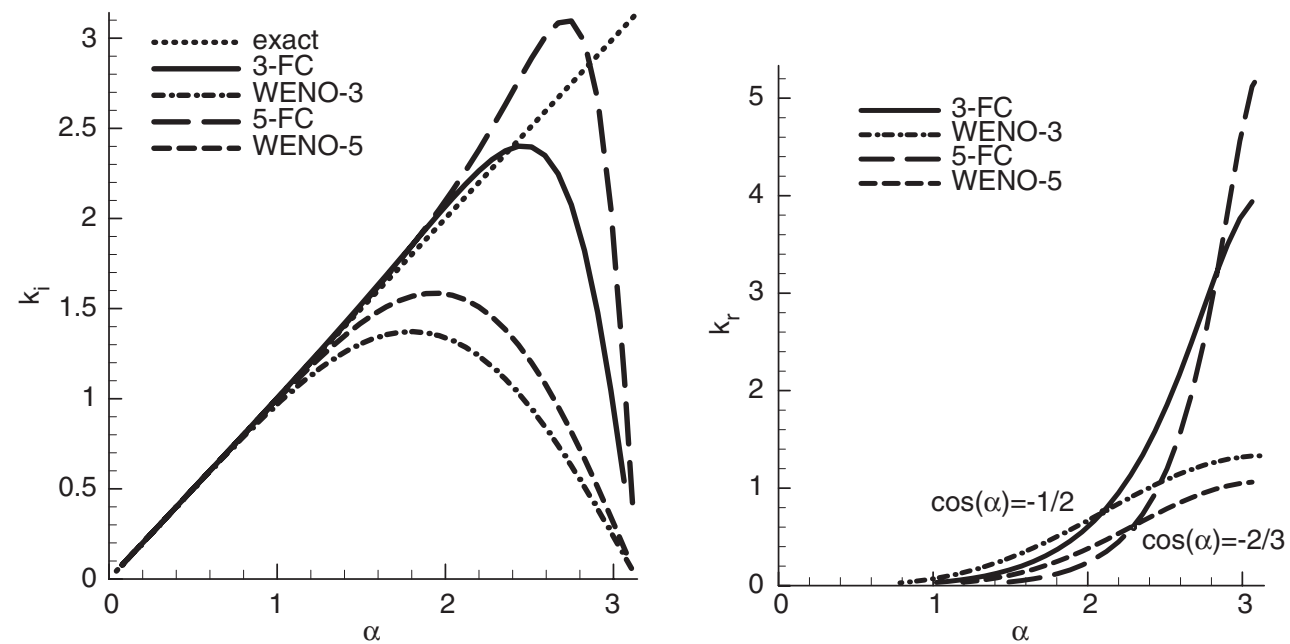

Figure 1. Variations of $k_{i}$ and $k_{r}$ of different schemes vs $\alpha$. 
only if $\alpha>\alpha_{c 2}\left(\cos \left(\alpha_{c 2}\right)=-2 / 3\right)$. Figure 1 also shows that the higher-order schemes (the FCschemes or the WENO schemes) produce better resolution properties.

Therefore, it is preferential to use the FC-schemes in smooth regions. At the same time, the WENO scheme yields better properties than its base ENO scheme. Based on the two reasons, the hybrid FC-WENO schemes are constructed.

\subsection{The hybrid finite compact-WENO schemes}

The scheme, which consists of the third-order finite compact scheme Equation (6), (4) with the modified limiter function

$$
\overline{\overline{m m}}\left(a_{1}, a_{2}, a_{3}\right)= \begin{cases}a_{1} & \text { if }\left|a_{1}\right| \leqslant \min \left(\left|a_{2}\right|,\left|a_{3}\right|\right) \\ a_{i} / 2 & \text { else if }\left|a_{i}\right|=\min \left(\left|a_{2}\right|,\left|a_{3}\right|\right)\end{cases}
$$

will be referred to as '3-FC-ENO-2'. The difference between $m m\left(a_{1}, a_{2}, a_{3}\right)$ and $\overline{\overline{m m}}\left(a_{1}, a_{2}, a_{3}\right)$ is that there is no fourth value (i.e. zero) in the latter.

In the same way, the hybrid FC-WENO scheme constructed by third-order FC-scheme coupled with third-order WENO scheme will be referred to as '3-FC-WENO-3', and its numerical fluxes' functions can be calculated by

$$
\begin{aligned}
& h_{j+1 / 2}^{+}= \begin{cases}\hat{h}_{j+1 / 2}^{+3-\mathrm{FC}} & \text { if }\left|\mathrm{d} f_{j+1 / 2}^{+}\right| \leqslant \min \left(\left|\Delta_{+} f_{j}^{+}\right|,\left|\Delta_{+} f_{j-1}^{+}\right|\right) \\
h_{j+1 / 2}^{+\mathrm{WENO}-3} & \text { otherwise }\end{cases} \\
& h_{j+1 / 2}^{-}= \begin{cases}\hat{h}_{j+1 / 2}^{-3-\mathrm{FC}} & \text { if }\left|\mathrm{d} f_{j+1 / 2}^{-}\right| \leqslant \min \left(\left|\Delta_{+} f_{j}^{-}\right|,\left|\Delta_{+} f_{j+1}^{-}\right|\right) \\
h_{j+1 / 2}^{-\mathrm{WENO}-3} & \text { otherwise }\end{cases}
\end{aligned}
$$

Similar to the analysis in Reference [22], the following equations are always satisfied:

$$
\mathrm{d} f_{j+1 / 2}^{ \pm}=\frac{1}{2} \frac{\partial f_{j}^{ \pm}}{\partial x} \Delta x+O\left(\Delta x^{2}\right), \quad \Delta_{+} f_{j}^{ \pm}=\frac{\partial f_{j}^{ \pm}}{\partial x} \Delta x+O\left(\Delta x^{2}\right)
$$

So in smooth regions away from critical points $\left(\partial f_{j}^{ \pm} / \partial x=0\right)$, formulas $\left|\mathrm{d} f_{j+1 / 2}^{+}\right| \leqslant \min \left(\left|\Delta_{+} f_{j}^{+}\right|\right.$, $\left.\left|\Delta_{+} f_{j-1}^{+}\right|\right)$and $\left|\mathrm{d} f_{j+1 / 2}^{-}\right| \leqslant \min \left(\left|\Delta_{+} f_{j}^{-}\right|,\left|\Delta_{+} f_{j+1}^{-}\right|\right)$are always satisfied. Therefore, for sufficiently small $\Delta x$, Equation (16) yields

$$
h_{j+1 / 2}^{ \pm}=\hat{h}_{j+1 / 2}^{ \pm 3-\mathrm{FC}}
$$

At critical points, the fluxes may become the fluxes of WENO-3 scheme, which is also third-order accurate. So 3-FC-WENO-3 Equation (16) is uniformly third-order accurate in smooth regions, and also makes the most of finite compact scheme.

Similarly, '5-FC-ENO-3' denotes the original scheme Equation (11). '5-FC-WENO-5' denotes the hybrid FC-WENO scheme, in which the fifth-order FC-scheme coupled with the fifth-order WENO scheme, and its numerical fluxes functions can be calculated as follows. The positive 
part is

$$
\begin{aligned}
& \text { if }\left|\Delta_{+} f_{j-1}^{+}\right| \leqslant\left|\Delta_{+} f_{j}^{+}\right| \text {then } \\
& h_{j+1 / 2}^{+}= \begin{cases}\hat{h}_{j+1 / 2}^{+5-\mathrm{FC}} & \text { if }\left|D f_{j+1 / 2}^{+}\right| \leqslant \min \left(\left|D_{j}^{+}\right|,\left|D_{j-1}^{+}\right|\right) \\
h_{j+1 / 2}^{+ \text {WENO-5 }} & \text { otherwise }\end{cases}
\end{aligned}
$$

else

$$
h_{j+1 / 2}^{+}= \begin{cases}\hat{h}_{j+1 / 2}^{+5-\mathrm{FC}} & \text { if }\left|D f_{j+1 / 2}^{+}\right| \leqslant \min \left(\left|D_{j}^{+}\right|,\left|D_{j+1}^{+}\right|\right) \\ h_{j+1 / 2}^{+ \text {WENO-5 }} & \text { otherwise }\end{cases}
$$

end if

and the negative part is

$$
\begin{aligned}
& \text { if }\left|\Delta_{+} f_{j+1}^{-}\right| \leqslant\left|\Delta_{+} f_{j}^{-}\right| \text {then } \\
& h_{j+1 / 2}^{-}= \begin{cases}\hat{h}_{j+1 / 2}^{-5-\mathrm{FC}} & \text { if }\left|D f_{j+1 / 2}^{-}\right| \leqslant \min \left(\left|D_{j+1}^{-}\right|,\left|D_{j+2}^{-}\right|\right) \\
h_{j+1 / 2}^{- \text {WENO-5 }} & \text { otherwise }\end{cases}
\end{aligned}
$$

else

$$
h_{j+1 / 2}^{-}= \begin{cases}\hat{h}_{j+1 / 2}^{-5-\mathrm{FC}} & \text { if }\left|D f_{j+1 / 2}^{-}\right| \leqslant \min \left(\left|D_{j}^{-}\right|,\left|D_{j+1}^{-}\right|\right) \\ h_{j+1 / 2}^{- \text {WENO-5 }} & \text { otherwise }\end{cases}
$$

end if

Similarly, there are

$$
\begin{aligned}
D f_{j+1 / 2}^{+}= \begin{cases}-\frac{1}{6} f_{j}^{+\prime} \Delta x^{2}+O\left(\Delta x^{3}\right) & \text { if }\left|\Delta_{+} f_{j}^{+}\right| \leqslant\left|\Delta_{+} f_{j-1}^{+}\right| \\
\frac{1}{3} f_{j}^{+\prime \prime} \Delta x^{2}+O\left(\Delta x^{3}\right) & \text { otherwise }\end{cases} \\
D f_{j+1 / 2}^{-}= \begin{cases}-\frac{1}{6} f_{j+1}^{-\prime \prime} \Delta x^{2}+O\left(\Delta x^{3}\right) & \text { if } \Delta_{+} f_{j+1}^{-}|\leqslant| \Delta_{+} f_{j}^{-} \mid \\
\frac{1}{3} f_{j+1}^{-\prime \prime} \Delta x^{2}+O\left(\Delta x^{3}\right) & \text { otherwise }\end{cases} \\
D_{k}^{+}=f_{j}^{+^{\prime \prime} \Delta x^{2}+O\left(\Delta x^{3}\right),} \quad k=j-1, j, j+1 \\
D_{k}^{-}=f_{j+1}^{-\prime \prime} \Delta x^{2}+O\left(\Delta x^{3}\right), \quad k=j, j+1, j+2
\end{aligned}
$$


With the same discussion as 3-FC-WENO-3, we know that the algorithm Equation (17) always yields $h_{j+1 / 2}^{ \pm}=\hat{h}_{j+1 / 2}^{ \pm 5-\mathrm{FC}}$ in the smooth regions away from point of $f_{j}^{ \pm^{\prime \prime}}=0$; and WENO-5 may be applied only at the point of $f_{j}^{ \pm^{\prime \prime}}=0$. So, whatever happens, 5-FC-WENO-5 is uniformly fifth-order accurate in smooth regions, and also makes the most of finite compact scheme.

\section{NUMERICAL EXAMPLES}

(1) Linear transport equation. The transport equation is given as

$$
\begin{gathered}
\frac{\partial u}{\partial t}+\frac{\partial u}{\partial x}=0, \quad-1 \leqslant x \leqslant 1 \\
u(x, 0)=\sin (\pi x), \quad-1 \leqslant x \leqslant 1, \text { periodic boundary condition }
\end{gathered}
$$

The example is used to examine the accuracy of different schemes. In our computation, a Courant number $\sigma=0.2$ is used. Table $\mathrm{V}$ gives the comparisons of accuracy of 3-FC-ENO-2, WENO3, 3-FC-WENO-3 and CEN4+WENOfi3 schemes. The table indicates that 3-FC-WENO-3 gives superior third-order accuracy and is the best one among 3-FC-ENO-2, WENO-3 and 3-FC-WENO-3 schemes. From the table, it seems that 3-FC-ENO-2 is less accurate than WENO-3, this is caused by the treatment of the critical points $\left(\partial f_{j}^{ \pm} / \partial x=0\right)$. In critical points, 3-FC-ENO-2 becomes second-order ENO scheme. In fact, in the regions away from critical points, 3-FC-ENO-2 is comparable to 3-FC-ENO-3 scheme. This can be seen from the comparisons of pointwise errors in Figure 2(a). One available treatment to overcome the drawback is to use a modified limiter function $\overline{m m}\left(a_{1}, a_{2}, a_{3}\right)$,

$$
\overline{m m}\left(a_{1}, a_{2}, a_{3}\right)= \begin{cases}a_{1} & \text { if }\left|a_{1}\right| \leqslant M \Delta x^{2} \\ m m\left(a_{1}, a_{2}, a_{3}\right) & \text { otherwise }\end{cases}
$$

to instead of $m m\left(a_{1}, a_{2}, a_{3}\right)$ in 3-FC-ENO-2, the discussion can be seen in Reference [22]. From Table V, we also can see that the 3-FC-ENO-3 is comparable to ACM with large parameter $k$ (e.g. $k=0.5$ ), but it is less accurate than ACM with small $k$ (e.g. $k=0.005)$.

Table VI gives the comparisons of accuracy of 5-FC-ENO-3, WENO-5, 5-FC-WENO-5 and CEN6+WENOfi5 schemes. The table indicates that 5-FC-WENO-5 gives fifth-order accuracy and is the best one among 5-FC-ENO-3, WENO-5 and 5-FC-WENO-5 schemes. CEN6+WENOfi5 with different parameter $k$ can reach sixth-order accuracy, and is better than 5-FC-WENO-5 schemes.

Similar to 3-FC-ENO-2 scheme, 5-FC-ENO-3 seems less accurate than WENO-5, this cause is that third-order ENO scheme is applied in points of $f_{j}^{ \pm^{\prime \prime}}=0$. In fact, in the regions away from points of $f_{j}^{ \pm \prime}=0,5$-FC-ENO-3 is in good agreement with the 5-FC-ENO-5 scheme (see Figure 2(b)).

That is to say, 5-FC-ENO-3 scheme is of fifth-order accurate in the regions away from points of $f_{j}^{ \pm^{\prime \prime}}=0$. The order degeneracy can be avoided by using the modified limiter functions 
Table V. Comparisons of different schemes, $t=1$.

\begin{tabular}{|c|c|c|c|c|c|}
\hline Scheme & $N$ & $L_{\infty}$ error & $L_{\infty}$ order & $L_{1}$ error & $L_{1}$ order \\
\hline \multirow{6}{*}{ 3-FC-WENO-3 } & 20 & 0.1024 & - & $0.4427 \mathrm{e}-1$ & - \\
\hline & 40 & $0.3833 e-1$ & 1.418 & $0.1068 \mathrm{e}-1$ & 2.051 \\
\hline & 80 & $0.1148 \mathrm{e}-1$ & 1.739 & $0.1748 \mathrm{e}-2$ & 2.611 \\
\hline & 160 & $0.1853 e-2$ & 2.631 & $0.1315 e-3$ & 3.733 \\
\hline & 320 & $0.5943 e-4$ & 4.963 & $0.3115 e-5$ & 5.400 \\
\hline & 640 & $0.1422 \mathrm{e}-5$ & 5.385 & $0.1614 \mathrm{e}-6$ & 4.271 \\
\hline \multirow{6}{*}{ WENO-3 } & 20 & 0.1205 & - & $0.5542 \mathrm{e}-1$ & - \\
\hline & 40 & $0.4794 \mathrm{e}-1$ & 1.330 & $0.1487 \mathrm{e}-1$ & 1.898 \\
\hline & 80 & $0.1716 \mathrm{e}-1$ & 1.482 & $0.3333 e-2$ & 2.158 \\
\hline & 160 & $0.4830 \mathrm{e}-2$ & 1.829 & $0.5779 \mathrm{e}-3$ & 2.528 \\
\hline & 320 & $0.8278 \mathrm{e}-3$ & 2.545 & $0.6222 \mathrm{e}-4$ & 3.215 \\
\hline & 640 & $0.5766 \mathrm{e}-4$ & 3.844 & $0.4010 e-5$ & 3.956 \\
\hline \multirow{6}{*}{ 3-FC-ENO-2 } & 20 & 0.1150 & --- & $0.5252 \mathrm{e}-1$ & --- \\
\hline & 40 & $0.4395 \mathrm{e}-1$ & 1.388 & $0.1283 e-2$ & 2.033 \\
\hline & 80 & $0.1573 e-1$ & 1.482 & $0.2757 \mathrm{e}-2$ & 2.218 \\
\hline & 160 & $0.5486 \mathrm{e}-2$ & 1.520 & $0.5557 \mathrm{e}-3$ & 2.307 \\
\hline & 320 & $0.1867 \mathrm{e}-2$ & 1.555 & $0.1097 \mathrm{e}-3$ & 2.344 \\
\hline & 640 & $0.6145 \mathrm{e}-3$ & 1.603 & $0.2055 \mathrm{e}-4$ & 2.416 \\
\hline \multirow{6}{*}{$\begin{array}{l}\text { ACM : } \\
\text { CEN4 + WENO3fi } \\
k=0.5\end{array}$} & 20 & $0.5882 \mathrm{e}-1$ & - & $0.2351 \mathrm{e}-1$ & - \\
\hline & 40 & $0.2316 \mathrm{e}-1$ & 1.345 & $0.6506 e-2$ & 1.853 \\
\hline & 80 & $0.7978 \mathrm{e}-2$ & 1.538 & $0.1433 e-2$ & 2.183 \\
\hline & 160 & $0.1812 \mathrm{e}-2$ & 2.138 & $0.2015 e-3$ & 2.830 \\
\hline & 320 & $0.1928 \mathrm{e}-3$ & 3.232 & $0.1166 \mathrm{e}-4$ & 4.111 \\
\hline & 640 & $0.8431 \mathrm{e}-5$ & 4.515 & $0.2678 \mathrm{e}-6$ & 5.444 \\
\hline \multirow{6}{*}{$\begin{array}{l}\text { ACM : } \\
\text { CEN4 + WENO3fi } \\
k=0.005\end{array}$} & 20 & $0.1115 \mathrm{e}-2$ & - & $0.6861 \mathrm{e}-3$ & - \\
\hline & 40 & $0.2109 \mathrm{e}-3$ & 2.402 & $0.7148 \mathrm{e}-4$ & 3.263 \\
\hline & 80 & $0.6801 \mathrm{e}-4$ & 1.633 & $0.1373 \mathrm{e}-4$ & 2.380 \\
\hline & 160 & $1.8885 \mathrm{e}-4$ & 1.851 & $0.2327 e-5$ & 2.561 \\
\hline & 320 & $0.2205 e-5$ & 3.100 & $0.1487 \mathrm{e}-6$ & 3.968 \\
\hline & 640 & $0.9311 \mathrm{e}-7$ & 4.566 & $0.3617 \mathrm{e}-8$ & 5.361 \\
\hline
\end{tabular}

$\overline{m m 1}\left(a_{1}, a_{2}, a_{3}\right)$ and $\overline{m m 2}\left(a_{1}, a_{2}, a_{3}\right)$,

$$
\begin{aligned}
& \overline{m m 1}\left(a_{1}, a_{2}, a_{3}\right)= \begin{cases}a_{1} & \text { if }\left|a_{1}\right| \leqslant M \Delta x^{3} \\
m m 1\left(a_{1}, a_{2}, a_{3}\right) & \text { otherwise }\end{cases} \\
& \overline{m m 2}\left(a_{1}, a_{2}, a_{3}\right)= \begin{cases}a_{1} & \text { if }\left|a_{1}\right| \leqslant M \Delta x^{3} \\
m m 2\left(a_{1}, a_{2}, a_{3}\right) & \text { otherwise }\end{cases}
\end{aligned}
$$

instead of $m m 1\left(a_{1}, a_{2}, a_{3}\right)$ and $m m 2\left(a_{1}, a_{2}, a_{3}\right)$ (see Reference [22]).

Comparing Figure 2(a) with (b), we can see that the large errors of different schemes locate at different region: 3-FC schemes locate at the regions including the maximum and 5-FC schemes locate at the regions including inflexion. This is agreement with the analysis of second-order ENO 

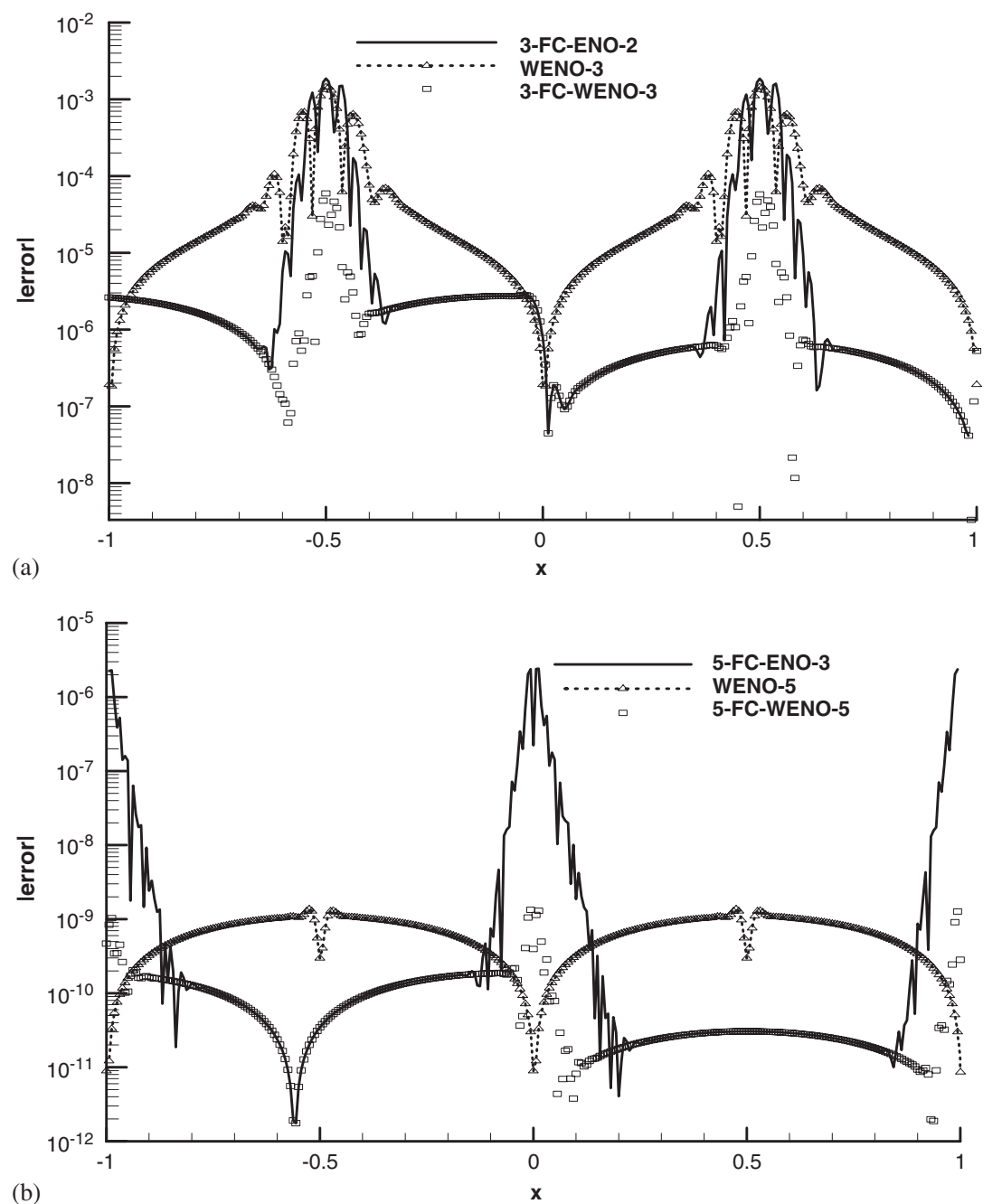

Figure 2. Pointwise errors of: (a) 3-FC-ENO-2, WENO-3 and 3-FC-WENO-3 schemes, $N=320, t=1$; and (b) 5-FC-ENO-3, WENO-5 and 5-FC-WENO-5 schemes, $N=320, t=1$.

and third-order ENO scheme are applied at critical points $\left(f_{j}^{ \pm^{\prime}}=0\right)$ and at points of $f_{j}^{ \pm^{\prime \prime}}=0$, respectively.

In order to compare the CPU and efficiency of different method, set $f(u)=u$ in Equation (1), we give the statistical times of multiplication, addition and logical operation in the computation of $h_{j+1 / 2}$ in Tables VII(a) and (b). Here, if both functions $\varphi_{j}=\varphi\left(u_{j+k}, \ldots, u_{j+p}\right)$ and $\varphi_{j+1}=\varphi\left(u_{j+1+k}, \ldots, u_{j+1+p}\right)$ appear in a formula, we only count the operations in $\varphi_{j}$. The multiplication of two constants is not regarded as one operation.

From Table VII(b), we can see, for the operations of multiplication and addition,WENO3+3RK is 16 and 27\% less than 3-FC-WENO-3+3RK; CEN4+WENO3fi+4RK is 31 and $7 \%$ less than 
Table VI. Comparisons of different schemes, $t=1$.

\begin{tabular}{|c|c|c|c|c|c|}
\hline Scheme & $N$ & $L_{\infty}$ error & $L_{\infty}$ order & $L_{1}$ error & $L_{1}$ order \\
\hline \multirow{6}{*}{ 5-FC-WENO-5 } & 10 & $0.7902 \mathrm{e}-2$ & - & $0.3897 \mathrm{e}-2$ & - \\
\hline & 20 & $0.3116 e-3$ & 4.664 & $0.1071 \mathrm{e}-3$ & 5.185 \\
\hline & 40 & $0.1379 \mathrm{e}-4$ & 4.498 & $0.3506 \mathrm{e}-5$ & 4.933 \\
\hline & 80 & $0.6282 \mathrm{e}-6$ & 4.456 & $0.1105 \mathrm{e}-6$ & 4.988 \\
\hline & 160 & $0.2897 \mathrm{e}-7$ & 4.439 & $0.3403 e-8$ & 5.021 \\
\hline & 320 & $0.1337 \mathrm{e}-8$ & 4.444 & $0.1048 \mathrm{e}-9$ & 5.021 \\
\hline \multirow{6}{*}{ WENO-5 } & 10 & $0.2958 \mathrm{e}-1$ & - & $0.1591 \mathrm{e}-1$ & - \\
\hline & 20 & $0.1455 \mathrm{e}-2$ & 4.346 & $0.7388 \mathrm{e}-3$ & 4.429 \\
\hline & 40 & $0.4592 \mathrm{e}-4$ & 4.986 & $0.2221 \mathrm{e}-4$ & 5.056 \\
\hline & 80 & $0.1478 \mathrm{e}-5$ & 4.957 & $0.6901 \mathrm{e}-6$ & 5.008 \\
\hline & 160 & $0.4417 \mathrm{e}-7$ & 5.064 & $0.2169 \mathrm{e}-7$ & 4.993 \\
\hline & 320 & $0.1367 \mathrm{e}-8$ & 5.014 & $0.6789 e-9$ & 4.999 \\
\hline \multirow{6}{*}{ 5-FC-ENO-3 } & 10 & $0.1548 \mathrm{e}-1$ & - & $0.7180 \mathrm{e}-2$ & - \\
\hline & 20 & $0.2576 \mathrm{e}-2$ & 2.587 & $0.7620 \mathrm{e}-3$ & 3.236 \\
\hline & 40 & $0.4588 \mathrm{e}-3$ & 2.489 & $0.8050 \mathrm{e}-4$ & 3.242 \\
\hline & 80 & $0.8497 \mathrm{e}-4$ & 2.433 & $0.8027 e-5$ & 3.326 \\
\hline & 160 & $0.1420 \mathrm{e}-4$ & 2.581 & $0.8187 \mathrm{e}-6$ & 3.293 \\
\hline & 320 & $0.2416 \mathrm{e}-5$ & 2.555 & $0.8264 \mathrm{e}-7$ & 3.308 \\
\hline \multirow{6}{*}{$\begin{array}{l}\text { ACM : } \\
\text { CEN6 + WENO5fi } \\
k=0.5\end{array}$} & 10 & $0.7119 \mathrm{e}-2$ & - & $0.5101 \mathrm{e}-2$ & - \\
\hline & 20 & $0.1912 \mathrm{e}-3$ & 5.219 & $0.9843 e-4$ & 5.696 \\
\hline & 40 & $0.6298 \mathrm{e}-5$ & 4.924 & $0.1772 \mathrm{e}-5$ & 5.796 \\
\hline & 80 & $0.1604 \mathrm{e}-6$ & 5.295 & $0.2965 \mathrm{e}-7$ & 5.901 \\
\hline & 160 & $0.3708 \mathrm{e}-8$ & 5.435 & $0.4767 e-9$ & 5.959 \\
\hline & 320 & $0.6849 \mathrm{e}-10$ & 5.789 & $0.6557 \mathrm{e}-11$ & 6.184 \\
\hline \multirow{6}{*}{$\begin{array}{l}\text { ACM : } \\
\text { CEN6 + WENO5fi } \\
k=0.005\end{array}$} & 10 & $0.1347 \mathrm{e}-2$ & - & $0.8713 e-3$ & - \\
\hline & 20 & $0.2210 \mathrm{e}-4$ & 5.930 & $0.1387 \mathrm{e}-4$ & 5.973 \\
\hline & 40 & $0.3540 \mathrm{e}-6$ & 5.964 & $0.2187 \mathrm{e}-6$ & 5.987 \\
\hline & 80 & $0.5664 \mathrm{e}-8$ & 5.966 & $0.3503 e-8$ & 5.964 \\
\hline & 160 & $0.9223 \mathrm{e}-10$ & 5.940 & $0.5841 \mathrm{e}-10$ & 5.906 \\
\hline & 320 & $0.1729 \mathrm{e}-11$ & 5.737 & $0.1087 \mathrm{e}-11$ & 5.748 \\
\hline
\end{tabular}

3-FC-WENO-3+3RK. WENO5+4RK is 13 and 22\% less than 5-FC-WENO-5+4RK; CEN6+ WENO5fi+4RK is 61 and $53 \%$ less than 5-FC-WENO-5+4RK.

For the one-dimensional Euler equation, if the Steger-Warming flux vector splitting [27] is used, we give the statistical computation of multiplication and addition of WENO5+4RK as

$$
(40 \times 4 \times 2+6) \times 3+45 \times 4=1158 ; \quad[(26+1) \times 4 \times 2+11] \times 3+25 \times 4=781
$$

where the factor 4 is the order of the Runge-Kutta method, the factor 2 denotes the flux splitting, and the factor 3 is the three conservative variables of the one-dimensional Euler equation. Other computations follow the similar idea. In the step of flux splitting, the operations include those in obtaining pressure from conservative variables; in CEN-methods, the operations include those in obtaining the fluxes from conservative variables. The comparisons are listed in Table VII(c). 
Table VII. Comparisons of the operations of different schemes and per time step.

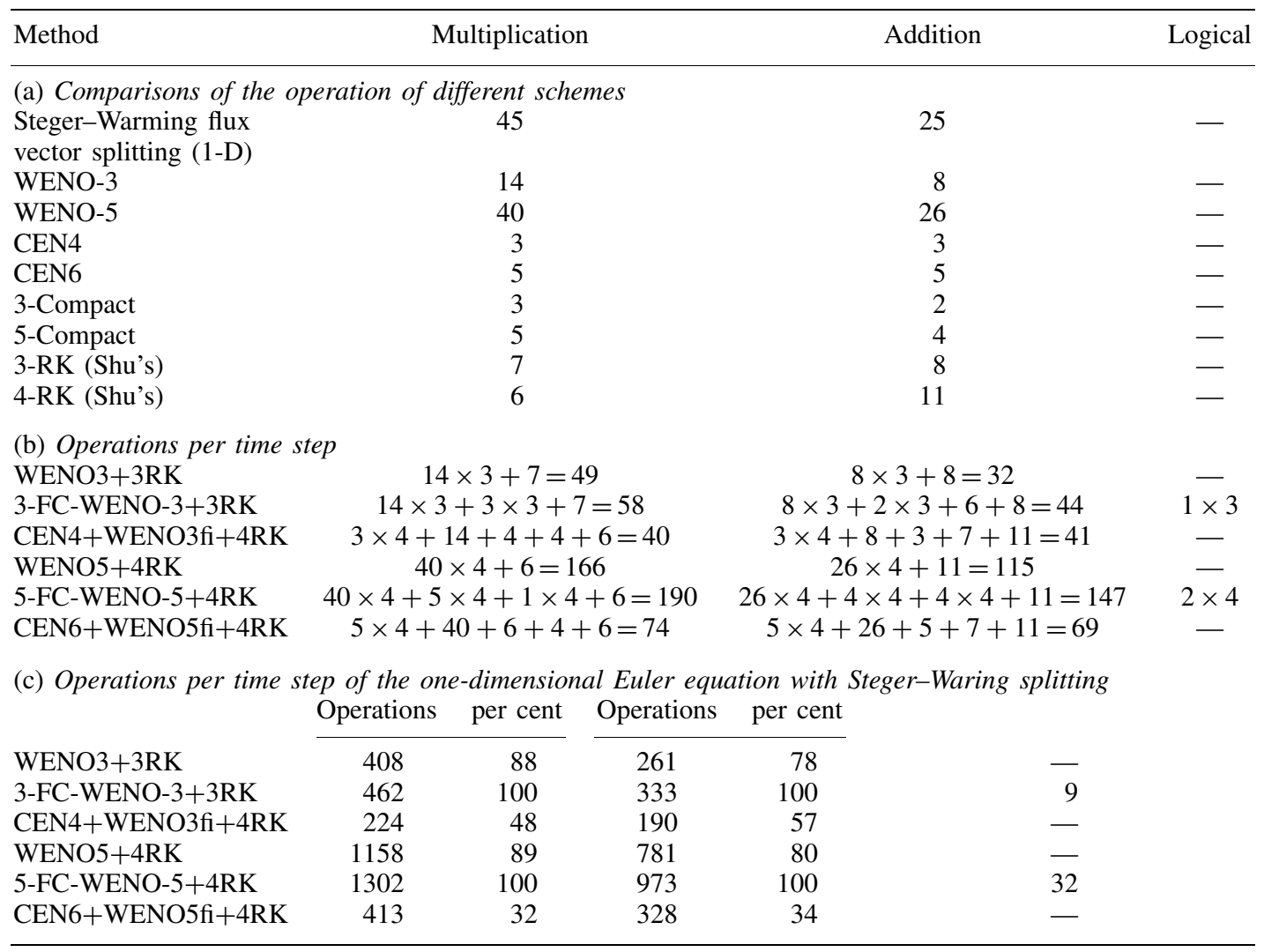

Table VII(c) shows that the WENOs only need 80\% CPU of FC-WENOs. CEN4+WENO3fi $+4 \mathrm{RK}$ only need about half CPU of 3-FC-WENO-3+3RK, and CEN6+WENO5fi+4RK only need about one-third CPU of 5-FC-WENO-5+4RK. So CEN4+WENO3fi+4RK is the most efficient one among WENO3+3RK, 3-FC-WENO-3+3RK and CEN4+WENO3fi+4RK; and CEN6+WENO5fi+4RK is the most efficient one among WENO5+4RK, 5-FC-WENO-5+4RK and CEN6+WENO5fi+4RK.

(2) $\partial u / \partial t+\partial u / \partial x=0,-1 \leqslant x \leqslant 1$. This equation is taken as the second test case where

$$
u(x+0.5,0)= \begin{cases}-x \sin \left(3 / 2 \pi x^{2}\right), & -1 \leqslant x<-1 / 3 \\ |\sin (2 \pi x)|, & |x|<1 / 3 \\ 2 x=-1-\sin (3 \pi x) / 6, & 1 / 3<x<1\end{cases}
$$

and supplemented with a periodic boundary condition. The example is used to check the capability of a numerical scheme to handle solutions with flow discontinuities and smooth regions at the same time $[2,19]$. The results are shown in Figures 3(a) and (f). Either for capturing discontinuities or for capturing smooth complex structures, FC-WENO schemes are the best ones, and FC-ENO schemes are better than WENO schemes. The results of the modified FC-ENO schemes, in which 

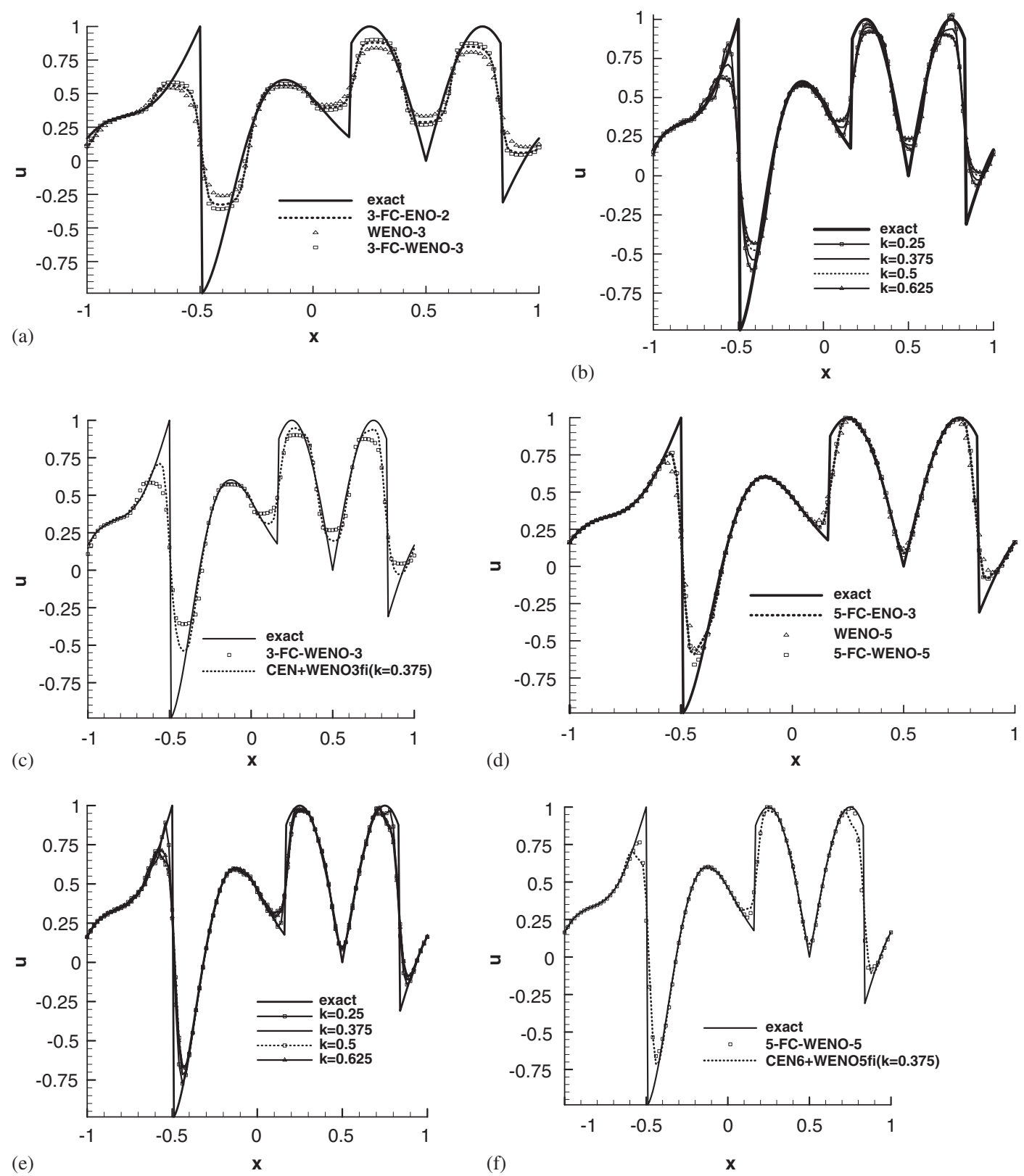

Figure 3. (a) Numerical results of case 2, $N=100, t=2$; (b) CEN4+WENO3fi with different $k$; (c) comparison of 3-FC-WENO-3 and CEN4+WENO3fi; (d) numerical results of case 2, $N=100, t=2$; (e) CEN6+WENO5fi with different $k$; and (f) comparison of 5-FC-WENO-5 and CEN6+WENO5fi. 
the modified limiter functions Equation (18) or Equation (19) with $M=1$ are used, are not depicted in the figure because of these are almost the same results as FC-ENO schemes.

From Figures 3(b) and (e), we can see that, if the solution contains discontinuity, the behaviour of artificial compression method is much affected by the problem-dependent parameter $k$. If $k$ is taken to too small, the solution will yield overshot or oscillation; if $k$ is taken to too large, the accuracy will degenerate. Figure 3(c) gives the comparison of 3-FC-WENO-3 and CEN4+WENO3fi with an appropriate parameter $k$ (here, $k=0.375$ ). It can be seen that CEN4+WENO3fi is better than 3-FC-WENO-3. Figure 3(f) gives the comparison of 5-FC-WENO-5 and CEN6+WENO5fi with an appropriate parameter $k$ (here, $k=0.375$ ). In the case, CEN6+WENO5fi is less accurate than 5-FC-WENO-5.

(3) $\partial u / \partial t+\partial u / \partial x=0,-1 \leqslant x \leqslant 1$. This equation is accepted as the third case where

$$
\begin{aligned}
& u(x, 0)= \begin{cases}\frac{1}{6}(G(x, \beta, z-\delta)+G(x, \beta, z+\delta)+4 G(x, \beta, z)), & -0.8 \leqslant x \leqslant-0.6 \\
1, & -0.4 \leqslant x \leqslant-0.2 \\
1-|10(x-0.1)|, & 0 \leqslant x \leqslant 0.2 \\
\frac{1}{6}(F(x, \alpha, a-\delta)+F(x, \alpha, a+\delta)+4 F(x, \alpha, a)), & 0.4 \leqslant x \leqslant 0.6 \\
0, & \text { otherwise }\end{cases} \\
& G(x, \beta, z)=\mathrm{e}^{-\beta(x-z)^{2},} \quad F(x, \alpha, a)=\sqrt{\max \left(1-\alpha^{2}(x-a)^{2}, 0\right),} \quad a=0.5, \quad z=-0.7 \\
& \delta=0.005, \quad \alpha=10, \quad \beta=\log 2 / 36 \delta^{2}
\end{aligned}
$$

The solution includes a smooth but narrow combination of Gaussians, a square wave, a sharp triangle wave, and a half-ellipse [5]. 200 grid points are used and the solution is intergraded up to $t=6$ in calculations. The results in Figure 4 indicate that the same conclusions as in second test case can be obtained for four types of waves.

Figures 4(b) and (f) give the results of ACMs with different parameter $k$. Figures 4(c) and (g) give the comparisons of FC-WENO schemes and ACMs. From Figure 4(c), it can be seen the result of Gaussians, the sharp triangle and the half-ellipse waves, CEN4+WENO3fi is more accurate than 3-FC-WENO-3, but for the square wave, 3-FC-WENO-3 is better. From Figure 4(g), 5-FC-WENO-5 is better than CEN6+WENO5fi $(k=0.375)$ for all four waves. We notice that for square wave, the results of all mentioned methods become asymmetrical. One reason is that the ENO schemes apply the upwind points, so do WENO schemes. The numerical results also can be seen Figure 2 in Reference [5], especially the result of WENO5 with artificial technique. Another reason of FC-schemes is that they apply the two-point upwind weighted compact schemes, Equation (6).

(4) Nonlinear transport equation. The nonlinear transport equation is used as the fourth test case and can be written as

$$
\begin{gathered}
\frac{\partial u}{\partial t}+u \frac{\partial u}{\partial x}=0, \quad 0 \leqslant x \leqslant 2 \pi \\
u(x, 0)=0.3+0.7 \sin x, \quad 0 \leqslant x \leqslant 2 \pi, \text { with a periodic boundary condition }
\end{gathered}
$$

The Lax-Friedrichs splitting method is used, in which $f^{ \pm}(u)=\frac{1}{2}(f(u) \pm a u)$ and $a=$ $\max _{u}\left|f^{\prime}(u)\right|$. Figures $5(\mathrm{a})$ and (b) show the results at $t=2$ with grid number of $N=80$. For 

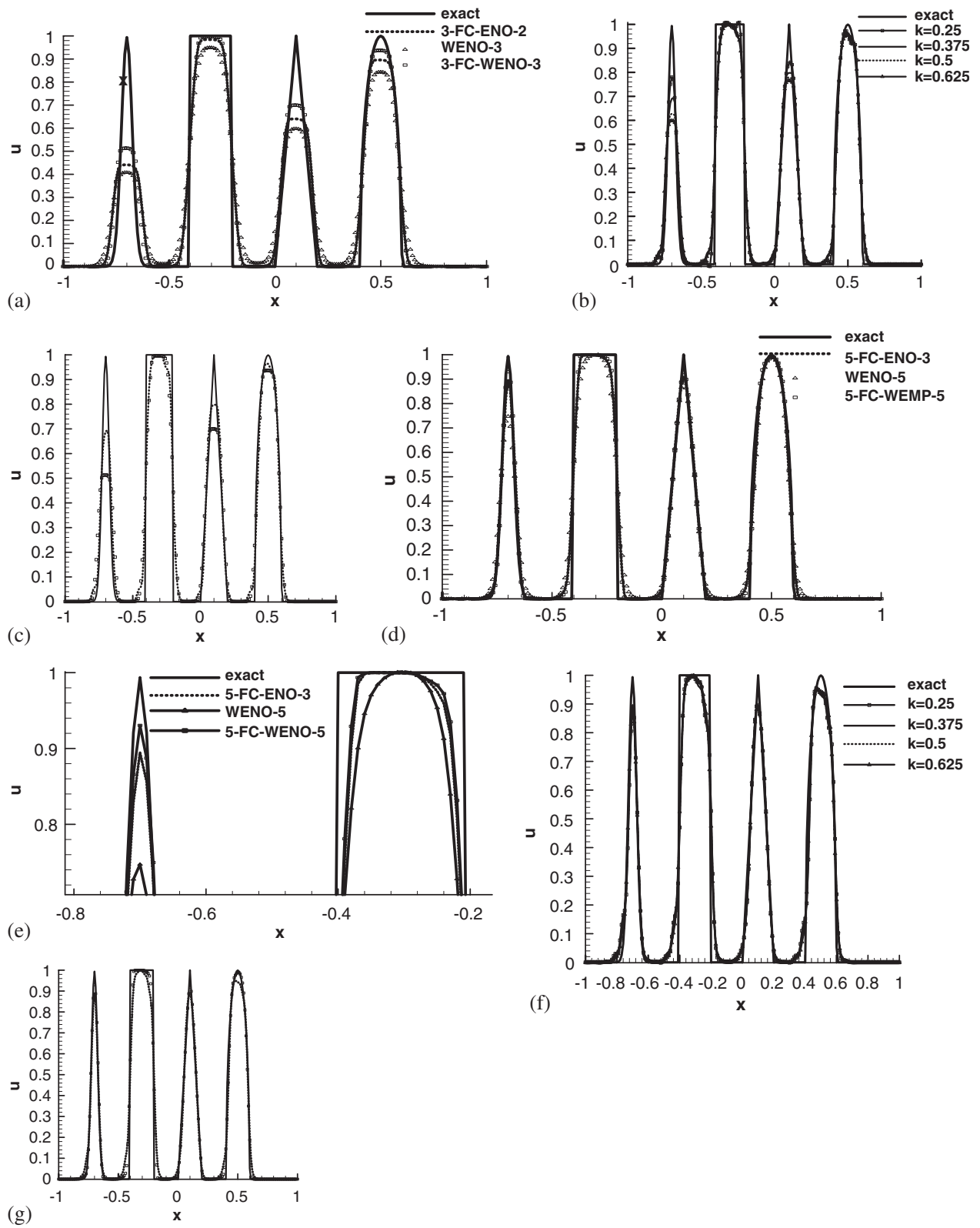

Figure 4. (a) Numerical results of example (3), $N=200, t=2$; (b) CEN4+WENO3fi with different $k$; (c) comparison of 3-FC-WENO-3 and CEN4+WENO3fi $(k=0.375)$; (d) numerical results of example (3), $N=200, t=6$; (e) the local enlarged part of Figure 4(b); (f) CEN6+WENO5fi with different $k$; and (g) comparison of 5-FC-WENO-5 and CEN6+WENO5fi $(k=0.375)$. 

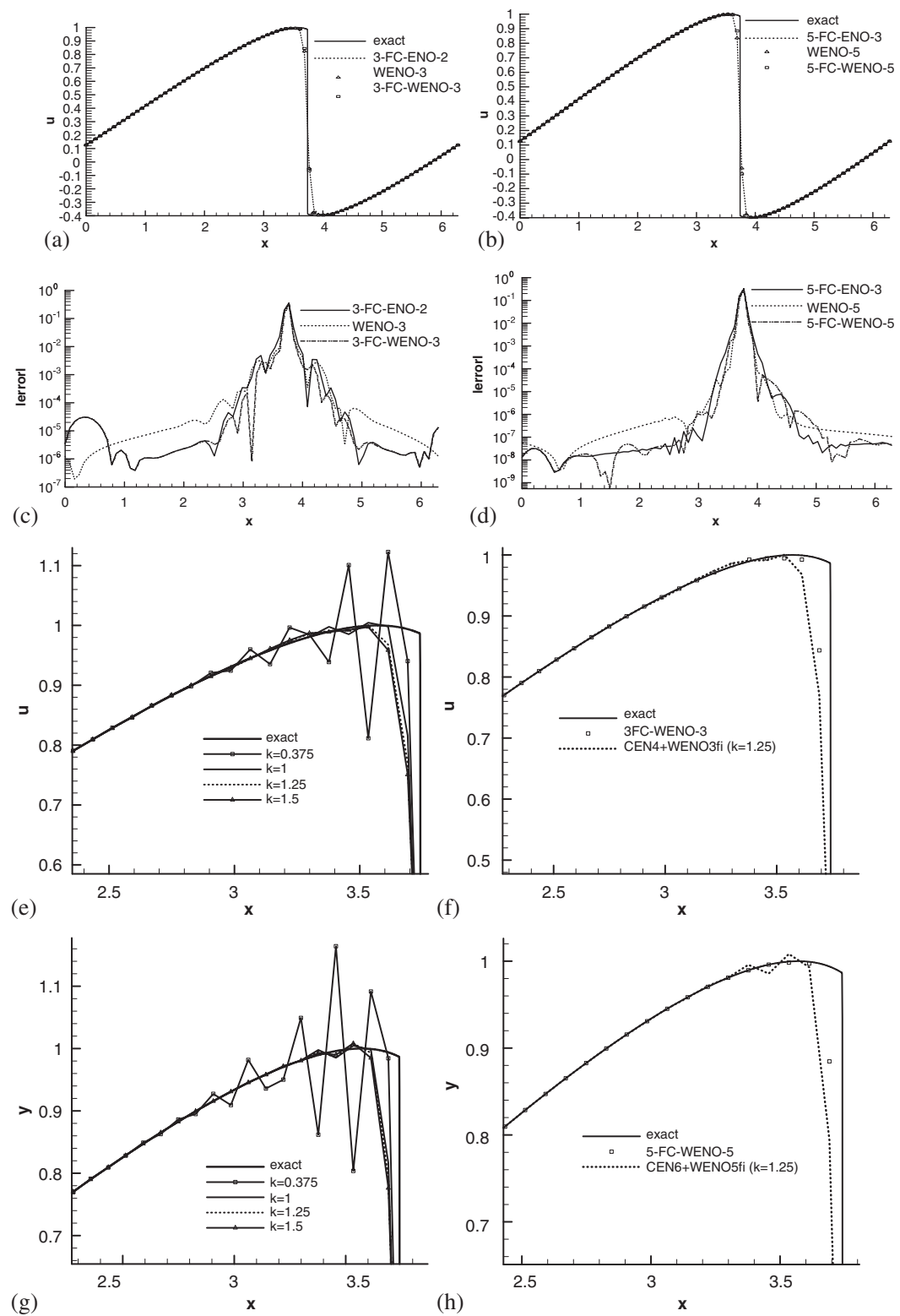

Figure 5. (a) Numerical results of example (4), $N=80, t=2$; (b) numerical results of example (4), $N=80, t=2$; (c) comparisons of pointwise errors, $N=80, t=2$; (d) comparisons of pointwise errors, $N=80, t=2$; (e) CEN4+WENO3fi with different $k$; (f) comparison of 3-FC-WENO-3 and CEN4+WENO3fi; (g) CEN6+WENO5fi with different $k$; and (h) comparison of 5-FC-WENO-5 and CEN6+WENO5fi. 
this case, the capability of capturing discontinuity of WENO is between FC-ENO and FC-WENO, and FC-WENO schemes are also the best ones. Figures 5(c) and (d) give the comparisons of pointwise errors. From Figures 5(c) and (d) we can see that in the region including discontinuity, FC-ENO, WENO, and FC-WENO are almost the same performance, but in the most regions away from the discontinuity, FC-ENO and FC-WENO obtain better accurate than WENO.

Figures 5(e) and (g) give the results of ACMs with different parameter $k$. Figures 5(f) and (h) give the comparisons of FC-WENO schemes and ACMs. Small parameter $k$ (e.g. $k=0.375$ ) causes the spurious oscillation near the discontinuity. Even with moderate $k$, the solution still has somewhat oscillation. From the comparisons, we can see the ACMs with $k=1.25$ are less than FC-WENO schemes for this case.

(5) Viscous Burgers equation. The viscous Burgers equation is our fifth case and written as

$$
\begin{aligned}
\frac{\partial u}{\partial t}+u \frac{\partial u}{\partial x} & =\frac{1}{\operatorname{Re}} \frac{\partial^{2} u}{\partial x^{2}}, \quad a \leqslant x \leqslant b \\
u(a) & =\tanh (-a \operatorname{Re} / 2) \\
u(b) & =\tanh (-b \operatorname{Re} / 2)
\end{aligned}
$$

The steady solution of Equation (21a) with boundary condition (21b) is $u(x)=\tanh (-x \operatorname{Re} / 2)$. At $x=0$, the shock is formed with a large Re number. The goal of this test case is to examine the capability of capturing the steady shock. In our computation, we take $a=-1$ and $b=1$, and the grid point number on $N=80$. The central difference scheme is applied for the secondorder derivative. The results with $R e=10^{2}$ and $R e=10^{3}$ are shown in Figures 6(a) and (b) (local enlarged). It can be seen that for the case with $R e=10^{3}$ resulting in strong shock, 3-FC-WENO-3 is almost the same as WENO-3; for the case with $R e=10^{2}$ resulting in weak shock, 3-FC-WENO3 is slightly better than WENO-3. FC-ENO-2 is less than 3-FC-WENO-3 and WENO-3. In the fifth-order schemes, 5-FC-WENO-5 is still best one, and in the case with strong shock, WENO-5 is better than 5-FC-ENO-3, however, in the case with weak shock, an opposite conclusion can be obtained.

Figures 6(c) and (e) give the results of ACMs with different parameter $k$. Figures 6(d) and (f) give the comparisons of FC-WENO schemes and ACMs. The behaviour of ACMs in this case is same as example (4). The results of the ACMs with $k=075$ are very near to FC-WENO schemes.

(6) Shu-Osher problem. Shu-Osher problem is the sixth example and it is governed with one-dimensional Euler equations

$$
\frac{\partial \mathbf{U}}{\partial t}+\frac{\partial \mathbf{F}}{\partial x}=0
$$

where $\mathbf{U}=(\rho, \rho u, \rho e)^{\mathrm{T}}, \mathbf{F}=\left(\rho u, \rho u^{2}+p, u(\rho e+p)\right)^{\mathrm{T}}, p=(\gamma-1)\left(\rho e-\rho u^{2} / 2\right), \gamma=1.4$. The initial condition is

$$
(\rho, u, p)= \begin{cases}(3.857143,2.629369,10.33333) & \text { when } x<-4 \\ (1+\varepsilon \sin 5 x, 0,1) & \text { when } x \geqslant-4\end{cases}
$$

Here, $\varepsilon=0.2$. This test case is taken from Reference [28], and has been studied by several other authors (see References [19, 20,22]), and represents a Mach 3 shock wave interacting with a sine entropy wave. The Steger-Warming flux vector splitting method [27] is used and the results at 

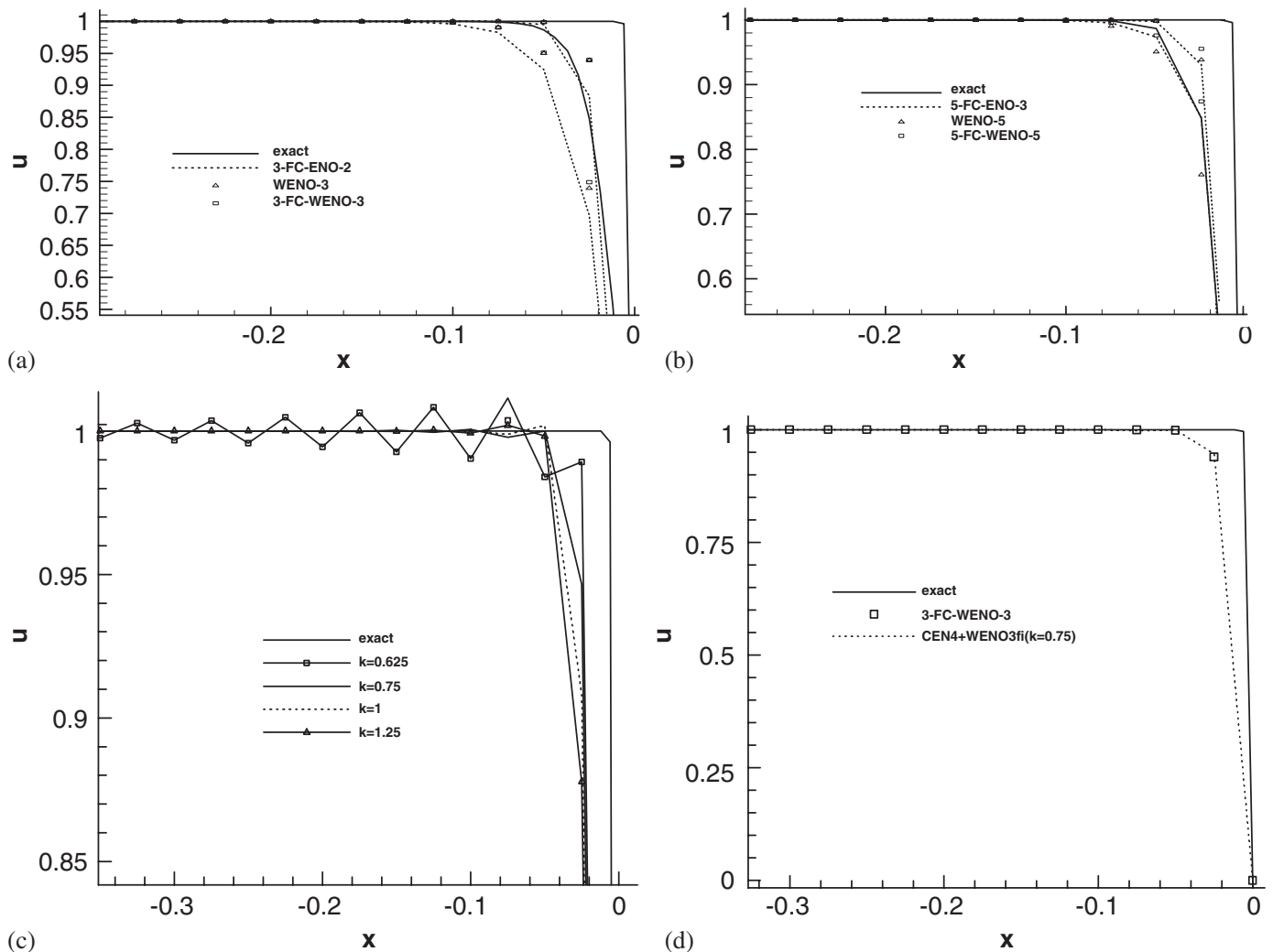

(c)

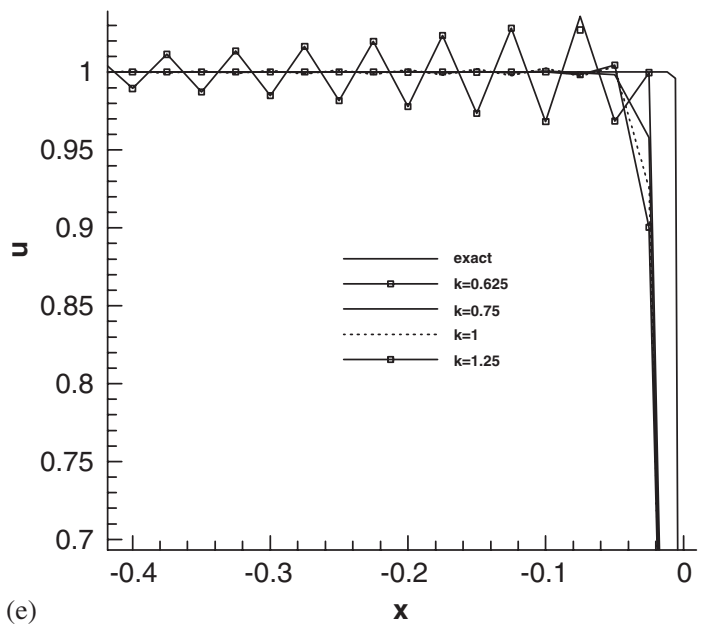

(d)

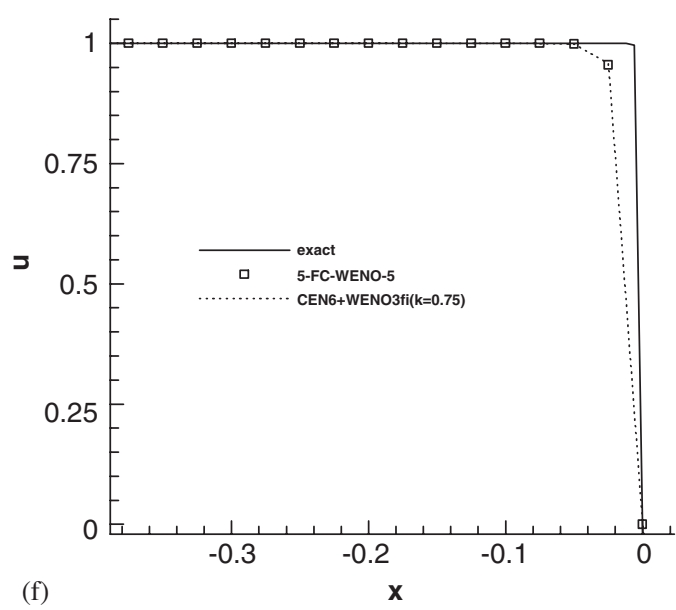

Figure 6. (a) Numerical results of steady solution of viscous Burgers equation; (b) numerical results of steady solution of viscous Burgers equation; (c) CEN4+WENO3fi with different $k$; (d) comparison of 3-FC-WENO-3 and CEN4+WENO3fi; (e) CEN6+WENO5fi with different $k$; and (f) comparison of 5-FC-WENO-5 and CEN6+WENO5fi. 

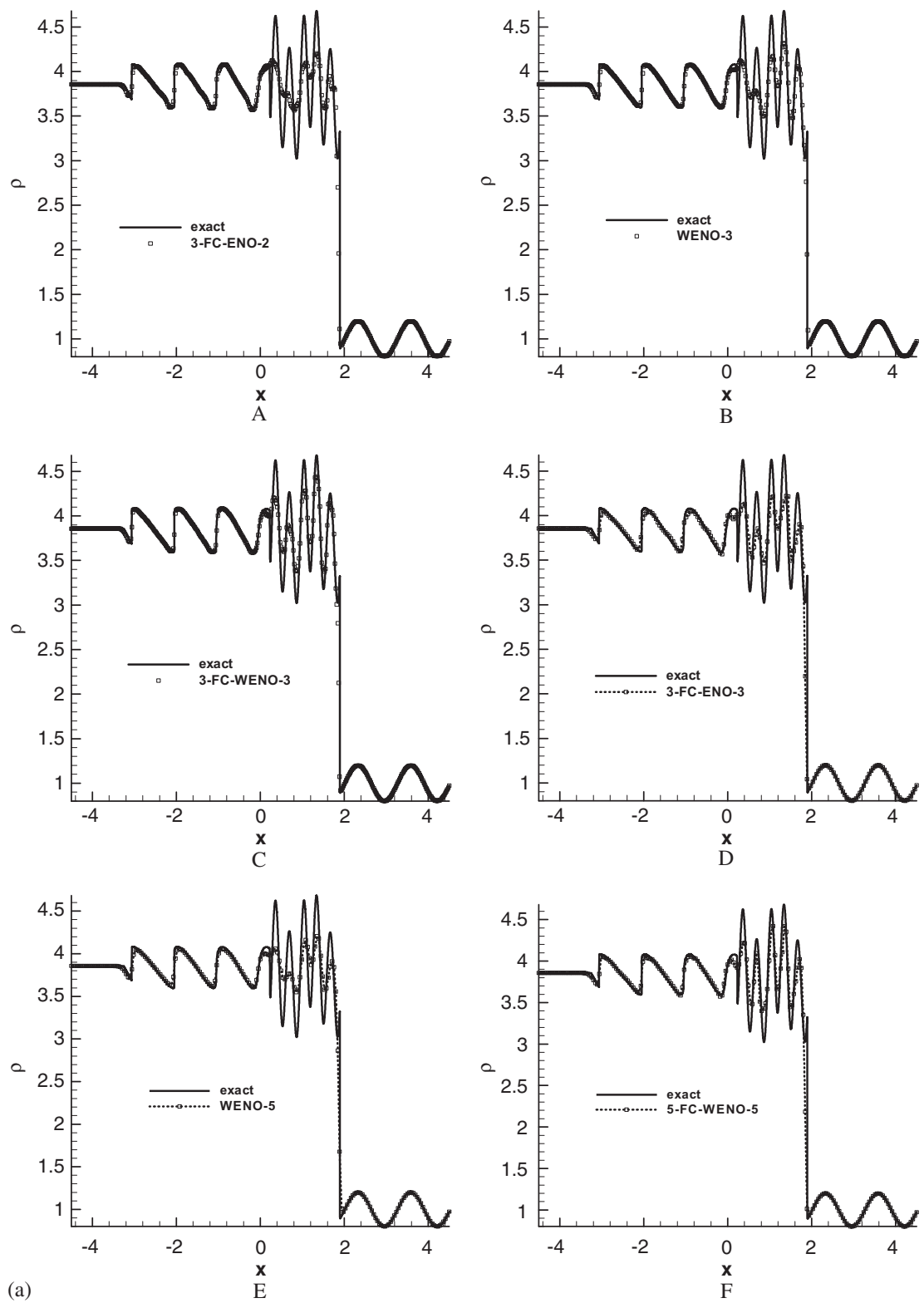

Figure 7. (a) Density distributions of Shu-Osher problem with $t=1.8$. Density distributions calculated by (A) 3-FC-ENO $(N=400)$, (B) WENO-3 $(N=400)$, (C) 3-FC-WENO-3 $(N=400)$, (D) 5-FC-ENO-3 $(N=200)$, (E) WENO-5 $(N=200)$, (F) 5-FC-WENO-5 $(N=200)$; (b) comparison of 3-FC-ENO-2, WENO-3 and 3-FC-WENO-3, $N=400$ (local enlarged); (c) comparison of 5-FC-ENO-3, WENO-5 and 5-FC-WENO-5, $N=200$ (local enlarged); (d) comparison of 3-FC-WENO-3 and CEN4+WENO3fi, $N=400$ (local enlarged); and (e) comparison of 5-FC-WENO-5 and CEN6+WENO5fi, $N=200$ (local enlarged). 
(b)

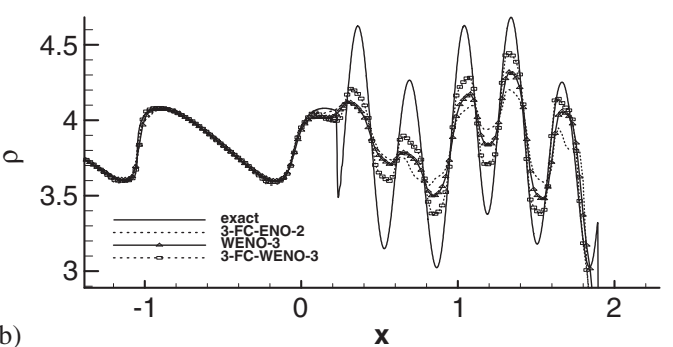

(d)

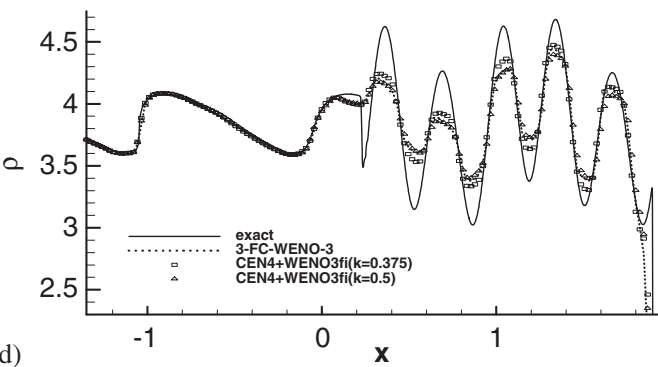

(c)
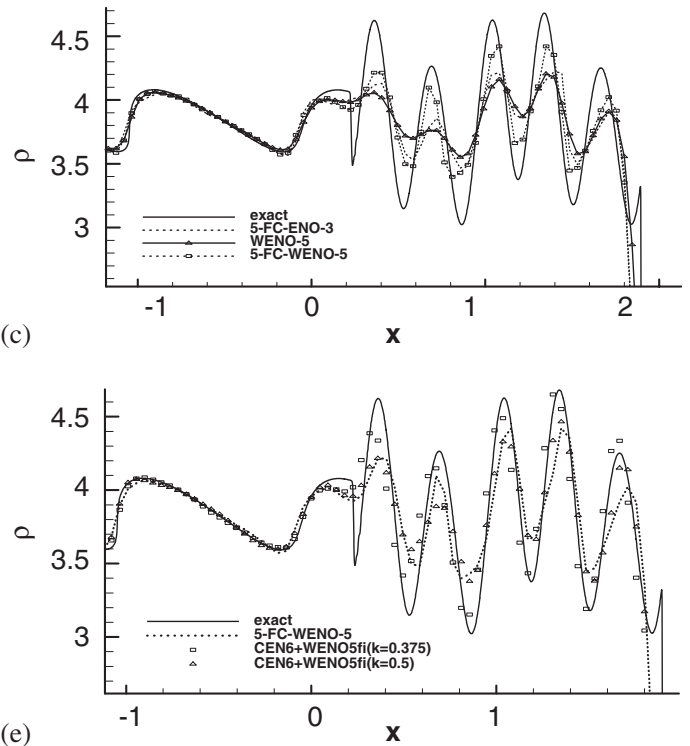

Figure 7. Continued.

time $t=1.8$ are plotted in Figures 7(a)-(c). The 'exact' solutions (solid lines) are the numerical solutions of WENO-5 scheme with grid points of $N=8000$. For this case, it can be shown that WENO-3 is between 3-FC-ENO-2 and 3-FC-WENO-3, 3-FC-WENO-3 has evidently improvement over WENO-3, see Figure 7(b). The result of 5-FC-WENO-5 is the best, and 5-FC-ENO-3 is also better than WENO-5, see Figure 7(c).

Figures 7(d) and (e) give the comparisons of FC-WENO schemes and ACMs. For this problem, the results of FC-WENO schemes are comparable to ACMs with $k=0.5$, and are less than ACMs with $k=0.375$. ACMs with smaller $k$ (e.g. $k=0.2$ ), the solution will become oscillatory.

(7) Two-dimensional linear conservation law with variable coefficients.

$$
\frac{\partial u}{\partial t}+\frac{\partial(-y u)}{\partial x}+\frac{\partial(x u)}{\partial y}=0, \quad-1 \leqslant x, y \leqslant 1
$$

with periodic boundary conditions. The initial condition is chosen as the characteristic function of a circle with radius 0.5 . The problem represents a solid body rotation and is used to investigate the grid orientation effect as in References [13, 22]. The results of 3-FC-WENO-3 and 5-FC-WENO5 at $t=2$ in a $100 \times 100$ points grid are shown in Figures $8(\mathrm{a})$ and (b). The numerical results indicate that the grid orientation effect is not strong. Figures 8(c) and (d) give the comparisons at $x=-0.02,-0.48$ and -0.52 , we can see that FC-WENO is the best one among FC-ENO and WENO, and FC-ENO is better than WENO, either the fifth-order scheme or the third-order scheme.

(8) Shock vortex interaction. The problem is taken from Reference [5]. It describes the interaction between a stationary shock and a vortex. The computational domain is taken to be $[0,2] \times[0,1]$ as in Reference [5]. A stationary Mach 1.1 shock is positioned at $x=0.5$ and normal to the $x$-axis. Its left state is $(\rho, u, v, P)=(1,1.1 \sqrt{\gamma}, 0,1)$. A small vortex is superposed to the flow left to 
(a)
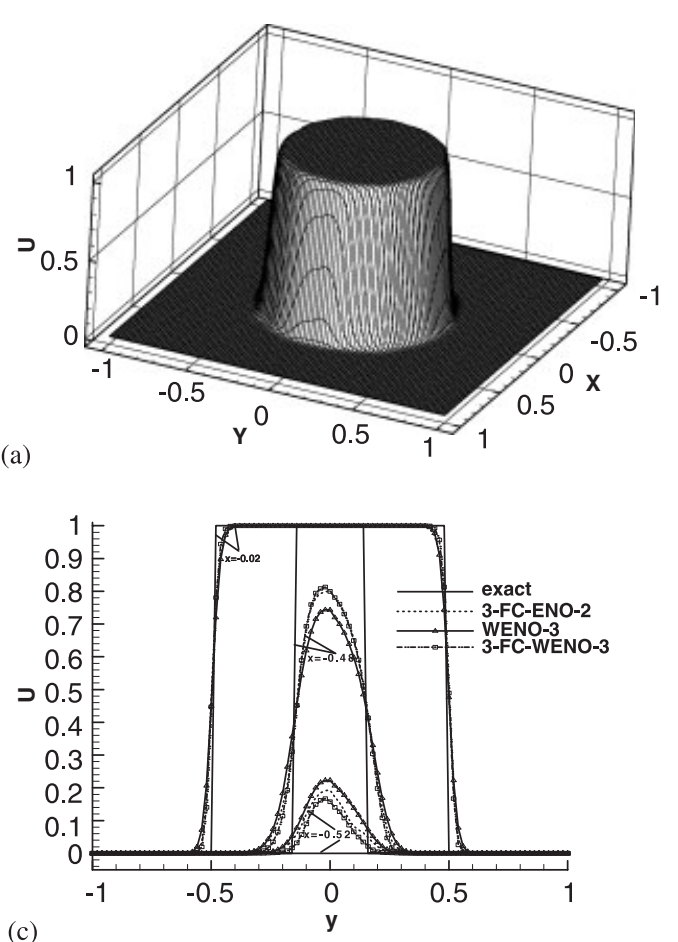

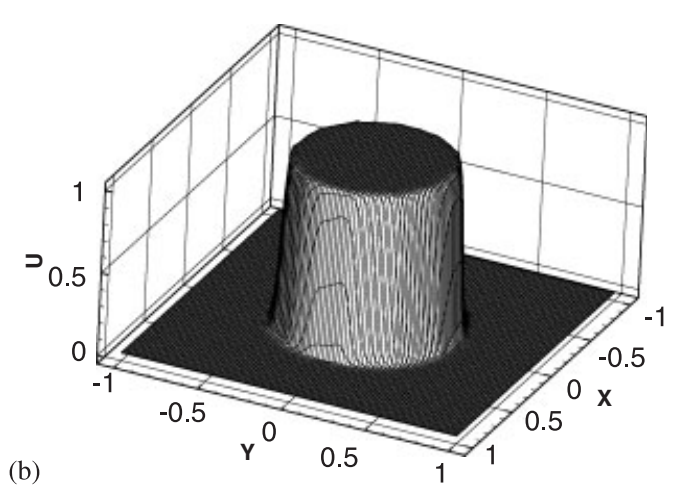

(b)

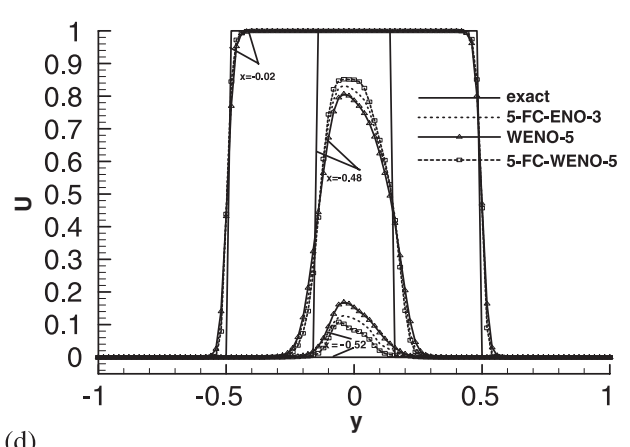

Figure 8. (a) Solution of 3-FC-WENO-3, $t=2$; (b) solution of 5-FC-WENO-5, $t=2$; (c) comparison at $x=-0.02,-0.48$ and -0.52 ; and (d) comparison at $x=-0.02,-0.48$ and -0.52 .

the shock and centres at $\left(x_{c}, y_{c}\right)=(0.25,0.25)$. The vortex is described as a perturbation to the velocity $(u, v)$, temperature $(T=P / \rho)$, and entropy $\left(S=\ln \left(P / \rho^{\gamma}\right)\right.$ of the mean flow and denoted by the tilde values:

$$
\begin{gathered}
\tilde{u}=\varepsilon \tau \mathrm{e}^{\alpha\left(1-\tau^{2}\right)} \sin \theta \\
\tilde{v}=-\varepsilon \tau \mathrm{e}^{\alpha\left(1-\tau^{2}\right)} \cos \theta \\
\widetilde{T}=-\frac{(\gamma-1) \varepsilon^{2} \mathrm{e}^{2 \alpha\left(1-\gamma^{2}\right)}}{4 \alpha \gamma} \\
\tilde{S}=0
\end{gathered}
$$

where $\tau=r / r_{c}$ and $r=\sqrt{\left(x-x_{c}\right)^{2}+\left(y-y_{c}\right)^{2}}$. Here $\varepsilon$ indicates the strength of the vortex, $\alpha$ controls the decay rate of the vortex, and $r_{c}$ is the critical radius for which the vortex has the maximum strength. $\varepsilon=0.3, r_{c}=0.05$, and $\alpha=0.204$.

We use a grid of $251 \times 101$ which is uniform in both $x$ and $y$ directions. The Steger-Warming flux vector splitting method [27] is used for this case. The 'exact' solution is computed by WENO-5 

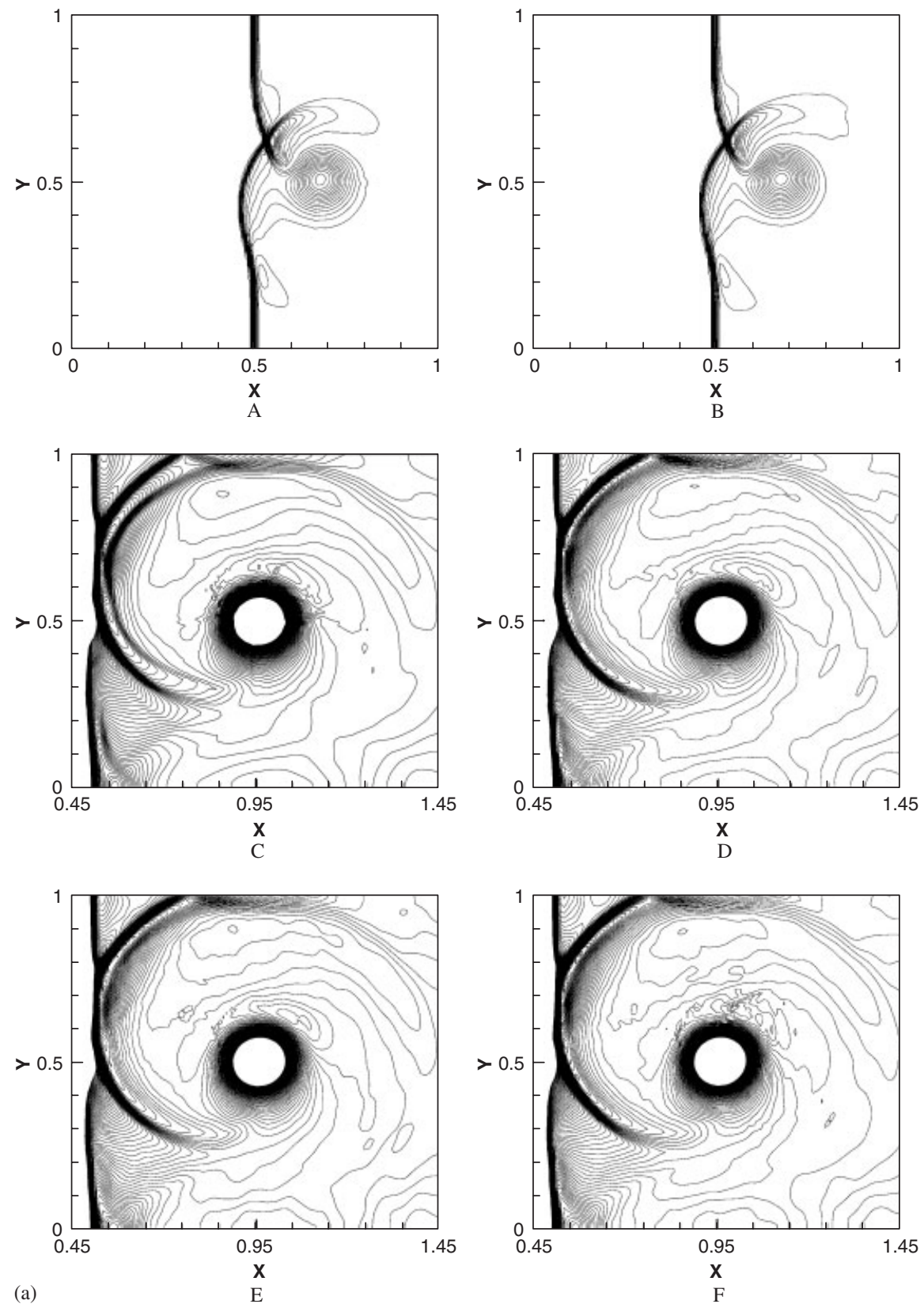

Figure 9. (a) Two-dimensional shock vortex interaction. Pressure. (A)-(B) $t=0.35$. Thirty contours: (A) 3-FC-WENO-3; (B) 5-FC-WENO-5; (C)-(F) $t=0.60$. Ninety contours: (C) 3-FC-WENO-3; (D) 5-FC-WENO-5; (E) WENO-5; (F) 5-FC-ENO-3; and (b) two-dimensional shock vortex interaction. Distribution of pressure along the $y=0.5$ section. $t=0.60$. (A) - (B) Comparison of 3-FC-ENO-2, WENO-3 and 3-FC-WENO-3;

(C)-(D) Comparison of 5-FC-ENO-3, WENO-5 and 5-FC-WENO-5. 

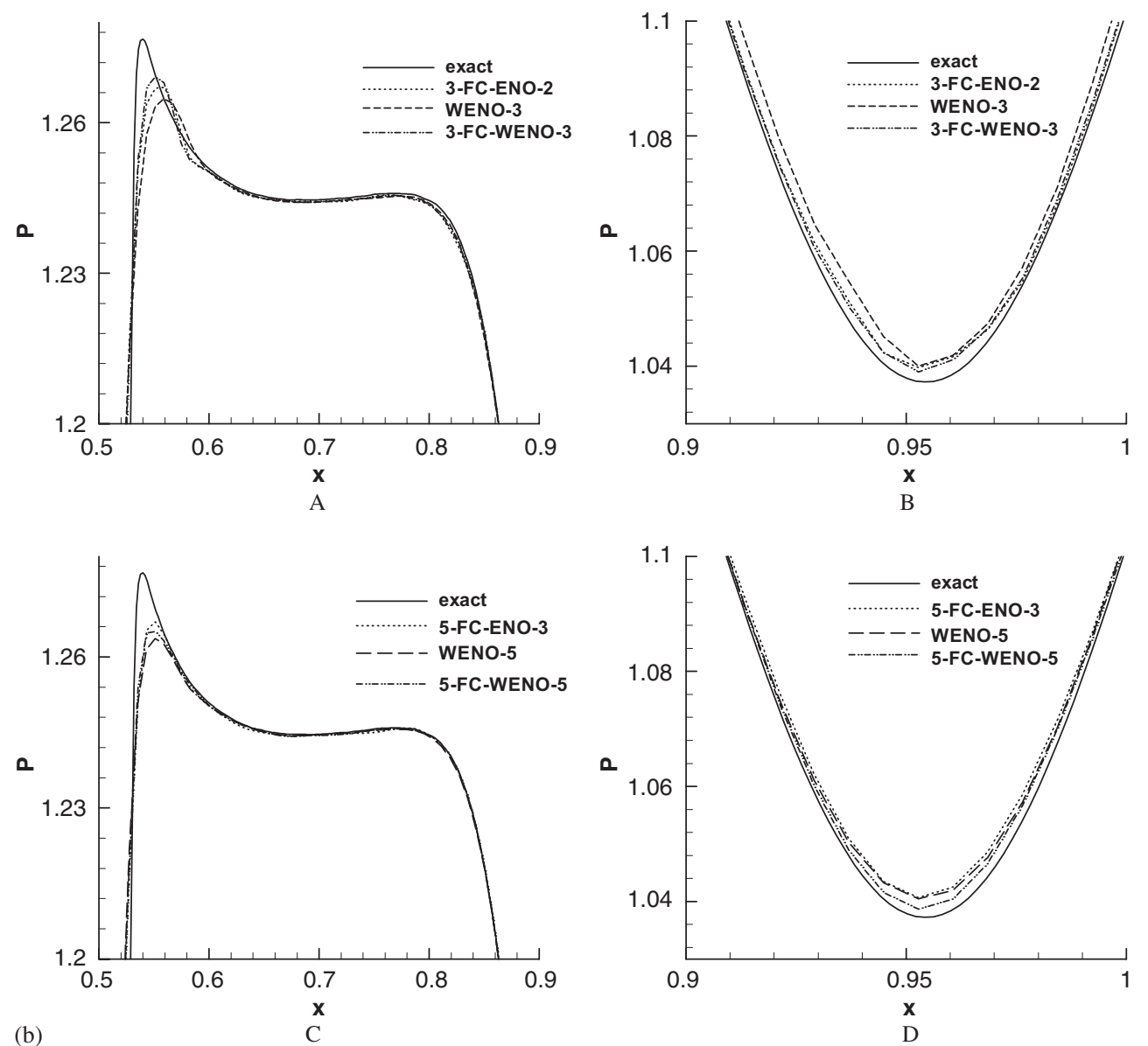

Figure 9. Continued.

scheme with a grid of $1000 \times 400$ nodes. The time step is taken as follows [19]:

$$
\Delta t=\sigma \frac{\Delta t_{x} \Delta t_{y}}{\Delta t_{x} \Delta t_{y}} \quad \text { with } \Delta t_{x}=\frac{\Delta x}{\max _{i, j}\left(\left|u_{i, j}\right|+c_{i, j}\right)}, \quad \Delta t_{y}=\frac{\Delta y}{\max _{i, j}\left(\left|v_{i, j}\right|+c_{i, j}\right)}, \quad \sigma=0.1
$$

The pressure contours are shown in Figure 9(a). The FC-ENO schemes, WENO schemes and FC-WENO schemes perform similarly. A careful comparing of the results obtained by 5-FCWENO-5, 5-FC-ENO-3 and WENO-5, we can find that 5-FC-WENO-5 and WENO-5 are slightly better than 5-FC-ENO-3 in the sense that less numerical noise is generated. Figure 9(b) gives the comparisons of distribution of pressure along the $y=0.5$ section. In the shock-behind and vortex-centre regions, somewhat better behaviours of FC-ENO and FC-WENO over WENO are observed. As a whole, FC-WENO is the best one.

(9) Shock/shear layer interaction. The problem is taken from Reference [7]. It is used to test the behaviour of the schemes for shock waves interacting with shear layers where the vortices arising from shear layer instability are forced to pass through a shock wave. 

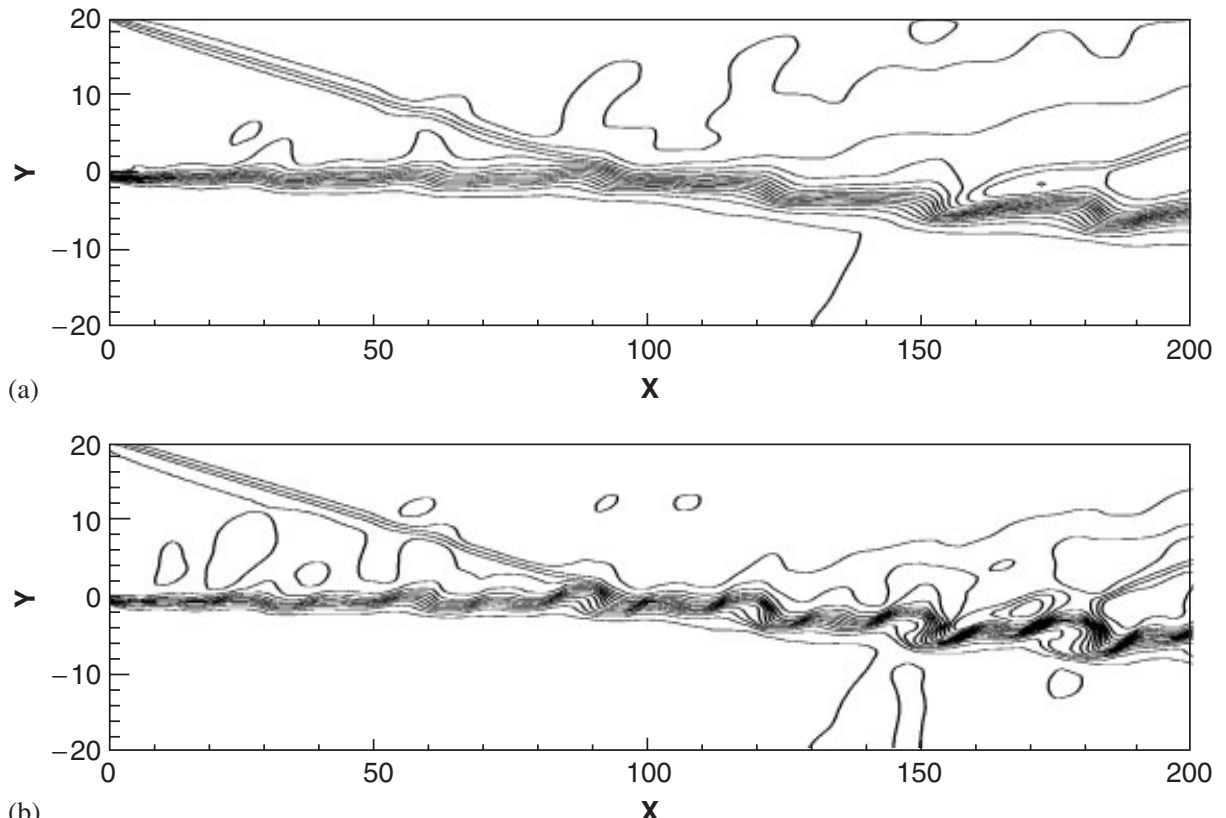

(b)

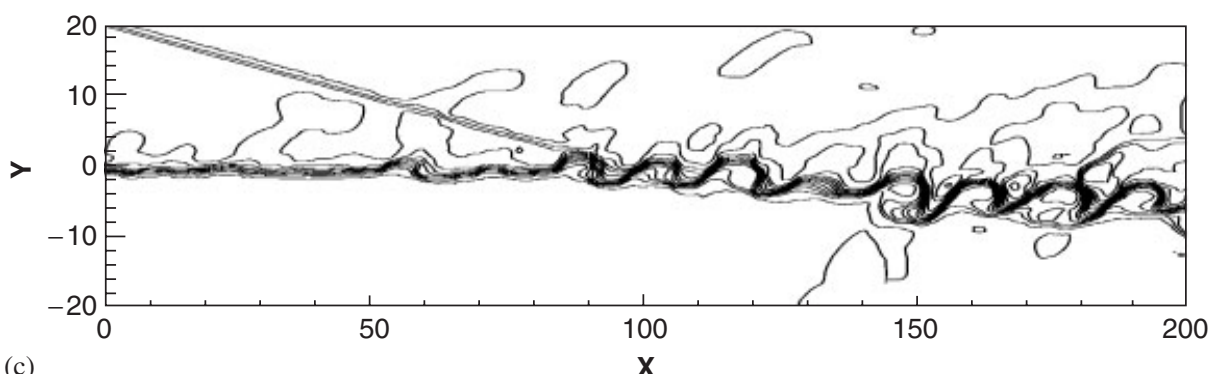

(c)

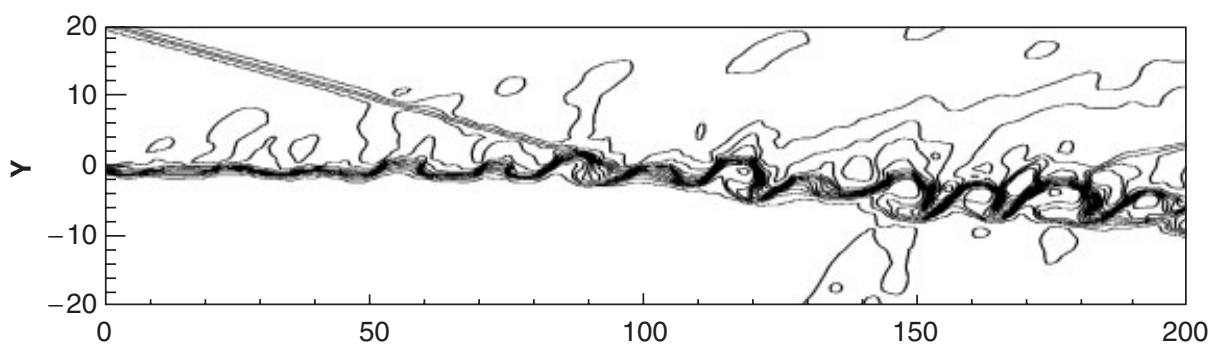

(d)

Figure 10. Density contours for shock/shear layer interaction at $t=120$ with a $321 \times 81$ grid: (a) density contours, TVD; (b) density contours, WENO-3; (c) density contours, 3-FC-ENO; (d) density contours, 3-FC-WENO-3; (e) density contours, ENO-3; (f) density contours, WENO-5; (g) density contours, 5-FC-ENO-3; and (h) density contours, 5-FC-WENO-5. 

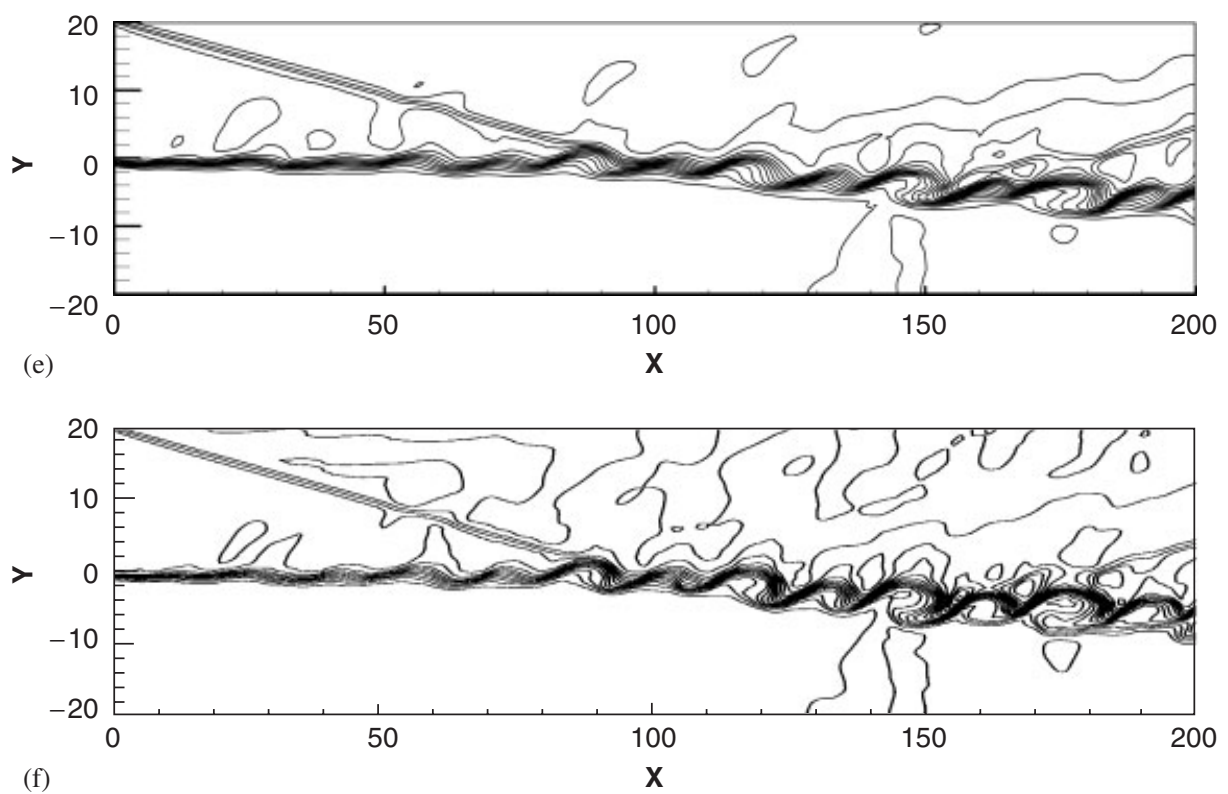

(f)

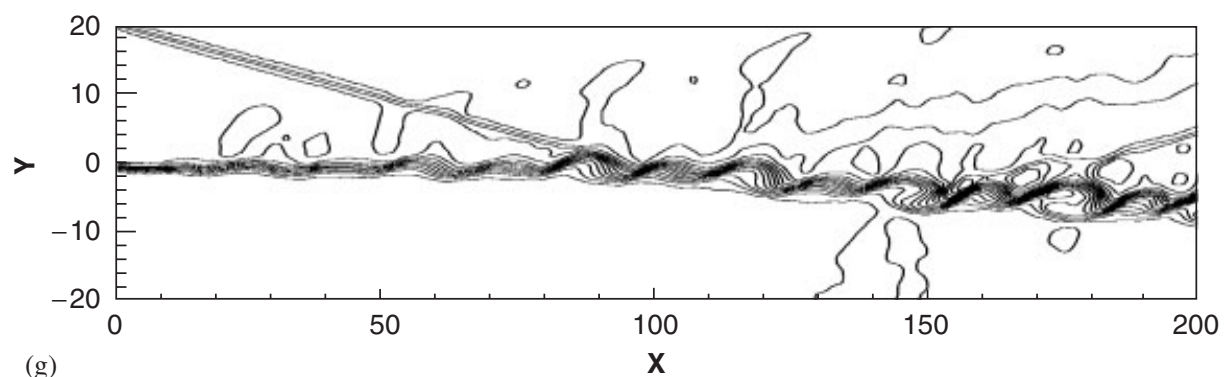

$(\mathrm{g})$

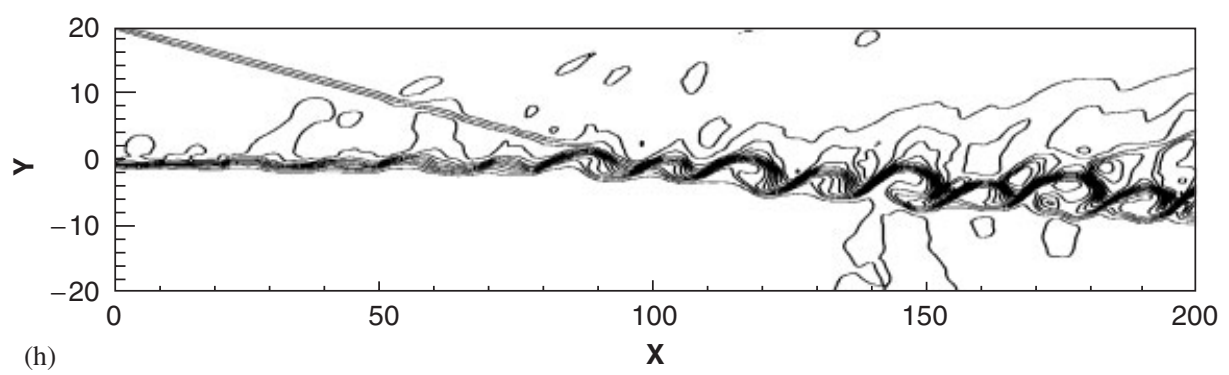

Figure 10. Continued.

An oblique shock with angle $\beta=12^{\circ}$ is made to impact on a spatially developing mixing layer at an initial convective Mach number of 0.5. The computation domain is taken to be $[x, y]=[0,200] \times[-20,20]$. The inflow is specified with a hyperbolic tangent profile,

$$
u=2.5+0.5 \tanh (2 y)
$$


For the upper stream inflow, $\rho_{u}=1.6374, p_{u}=0.3327$; for the lower stream inflow, $\rho_{l}=0.3626$, $p_{l}=0.3327$. The upper boundary condition is taken from the flow properties behind the oblique shock. The lower wall uses a slip condition.

Fluctuations are added to the $v$-component of velocity to the inflow as

$$
v^{\prime}=\sum_{k=1}^{2} a_{k} \cos \left(2 \pi k t / T+\phi_{k}\right) \exp \left(-y^{2} / b\right)
$$

with period $T=\lambda / u_{c}$, wavelength $\lambda=30$, convective velocity $u_{c}=2.68, b=10, a_{1}=a_{2}=0.05$, $\phi_{1}=0$ and $\phi_{2}=\pi / 2$.

The controlling equation is two-dimensional Navier-Stokes equation. The Prandtl number is set to 0.72 , and the Reynolds number is chosen to be 500 . The central difference scheme is used for the viscous terms. The same grid as in Reference [7], $321 \times 81$, is used. The time step is taken as Equation (22) with $\sigma=0.5$.

The density contours are shown in Figure 10. As the same result in Reference [7], the TVD scheme misses the correct vortex formation. WENO-3 does so. 3-FC-WENO-3 shows better result than 3-FC-ENO-3, and it is comparable to third-order ENO scheme. WENO-5 is better than 5-FC-ENO-3, and less than 5-FC-WENO-5. This example illustrates the FC-schemes are applicable for the complex two-dimensional shock/shear layer interaction flow.

\section{CONCLUSIONS}

Based on the analysis, that the finite compact difference scheme has better resolution of high wavenumbers than WENO scheme and the WENO scheme possesses better performances than its base ENO scheme, the hybrid finite compact (FC)-WENO schemes are proposed for shock calculations. The FC-WENO schemes can satisfy the requirement that the FC-schemes are used as possible in smooth region and the WENO schemes are used at the discontinuous point or critical point of $f_{j}^{ \pm^{\prime}}=0$ (WENO-3 is used in 3-FC-WENO-3) and at point of $f_{j}^{ \pm^{\prime \prime}}=0$ (WENO-5 is used in 5-FC-WENO-5). The numerical results show that FC-WENO schemes have the uniformly highorder accuracy, and can improve the capability of shock capturing and fine-scale feature capturing over the WENO schemes and the original developed FC-ENO schemes. The ACM filter schemes of Yee et al. have the higher efficiencies than FC-WENOs and WENOs, the disadvantage of them is that they need to decide an appropriate problem-dependent parameter $k$.

\section{ACKNOWLEDGEMENTS}

This work is supported by the National Natural Science Foundation of China (No. 10402043, 10372106).

\section{REFERENCES}

1. Harten A. High resolution schemes for hyperbolic conservation laws. Journal of Computational Physics 1983; 49:357-393.

2. Harten A, Engquist B, Osher S, Chakravarthy SR. Uniformly high order accuracy essentially non-oscillatory schemes. III. Journal of Computational Physics 1987; 71:231-303.

3. Shu CW, Osher S. Efficient implementation of essentially non-oscillatory shock-capturing schemes. II. Journal of Computational Physics 1989; 83:32-78. 
4. Liu XD, Osher S, Chan T. Weighted essentially non-oscillatory schemes. Journal of Computational Physics 1994; 115:200-212.

5. Jiang GS, Shu CW. Efficient implementation of weighted ENO schemes. Journal of Computational Physics 1996; 126:202-228.

6. Kim D, Kwon JH. A high-order accurate hybrid scheme using a central flux scheme and a WENO scheme for compressible flowfield analysis. Journal of Computational Physics 2005; 210:554-583.

7. Yee HC, Sandham ND, Djomehri MJ. Low-dissipative high-order shock capturing methods using characteristicbased filters. Journal of Computational Physics 1999; 150:199-238.

8. Sjogreen B, Yee HC. Grid convergence of high order methods for multiscale complex unsteady viscous compressible flows. Journal of Computational Physics 2003; 185:1-26.

9. Wang ZJ, Chen RF. Optimized weighted essentially non-oscillatory schemes for linear waves with discontinuity. Journal of Computational Physics 2001; 174:381-404.

10. Balsara D, Shu CW. Monotonicity preserving weighted essentially non-oscillatory schemes with increasingly high order of accuracy. Journal of Computational Physics 2000; 160:405-452.

11. Jiang Z. On dispersion-controlled principles for non-oscillatory shock-capturing schemes. Acta Mechanica Sinica 2004; $20(1): 1-15$.

12. Lele SK. Compact finite difference schemes with spectral-like resolution. Journal of Computational Physics 1992; 103:16-42.

13. Cockburn B, Shu CW. Nonlinearly stable compact schemes for shock calculations. SIAM Journal on Numerical Analysis 1994; 31(3):607-627.

14. Ravichandran KS. Higher order KFVS algorithms using compact upwind difference operators. Journal of Computational Physics 1997; 130:161-173.

15. Deng XG, Zhang HX. Developing high-order weighted compact nonlinear schemes. Journal of Computational Physics 2000; 165:22-44.

16. Zhu QY, Chan CK. An upwind compact approach with group velocity control for compressible flow fields. International Journal for Numerical Methods in Fluids 2004; 44:463-482.

17. Fu DX, Ma YW. A high order accurate finite difference scheme for complex flow fields. Journal of Computational Physics 1997; 134:1-15.

18. Adams NA, Shariff K. A high-resolution hybrid compact-ENO scheme for shock-turbulence interaction problems. Journal of Computational Physics 1996; 127:27-51.

19. Pirozzoli S. Conservative hybrid compact-WENO schemes for shock-turbulence interaction. Journal of Computational Physics 2002; 178:81-117.

20. Ren YX, Liu M, Zhang HX. A characteristic-wise hybrid compact-WENO scheme for solving hyperbolic conservation laws. Journal of Computational Physics 2003; 192:365-386.

21. Tolstykh AI, Lipavskii MV. On performance of methods with third- and fifth-order compact upwind differencing. Journal of Computational Physics 1998; 140:205-232.

22. Shen YQ, Yang GW, Gao Z. High-resolution finite compact difference schemes for hyperbolic conservation laws. Journal of Computational Physics 2006; 216:114-137.

23. Yee HC, Sjogreen B. Designing adaptive low dissipative high order schemes for long-time integrations. RIACS Technical Report TR01-28, December 2001; In Turbulent Flow Computation, Drikakis D, Geurts B (eds). Kluwer Academic Publishers: Dordrecht, MA, 2002; 141-198.

24. Yee HC, Sjogreen B. Nonlinear filtering in compact high order schemes. International Conference on Numerical Simulation of Plasma, 12-15 July, 2005, Nara, Japan; Journal of Plasma Physics, in press.

25. Carpenter MH, Gottlieb D, Abarbanel S. The stability of numerical boundary treatments for compact high-order finite-difference schemes. Journal of Computational Physics 1993; 108:272-295.

26. Ma YW, Fu DX, Kobayashi T, Taniguchi N. Numerical solution of the incompressible Navier-Stokes equations with an upwind compact difference scheme. International Journal for Numerical Methods in Fluids 1999; 30:509-521.

27. Steger JL, Warming RF. Flux vector splitting of the inviscid gasdynamic equations with application to finitedifference methods. Journal of Computational Physics 1981; 40:263-293.

28. Shu CW, Osher S. Efficient implementation of essentially non-oscillatory shock-capturing schemes. Journal of Computational Physics 1988; 77:439-471. 